\title{
Fluorine Conformational Effects Characterized by Energy Decomposition Analysis
}

Natalia Díaz, * Fernando Jiménez-Grávalos, Dimas Suárez, Evelio Francisco, and Ángel Martín-Pendás

Departamento de Química Física y Analítica. Universidad de Oviedo. Avda. Julián Clavería 8, 33006 Oviedo (Asturias). Spain.

diazfnatalia@uniovi.es

\begin{abstract}
Electrostatic and stereoelectronic effects associated to fluorine atoms can be exploited as conformational tools for the design of shape-controlled functional molecules. To gain further insight into the nature and strength of these effects, we use the Interacting Quantum Atoms (IQA) method augmented with the semiclassical pairwise dispersion potential to decompose the conformational energies of fluoro-substituted molecules into fragment-based energy contributions, which include deformation/distortion terms and the electrostatic, exchangecorrelation and dispersion interactions. The studied molecules comprise various $\mathrm{F}-\mathrm{CH}_{2}-\mathrm{CH}_{2}-$ $\mathrm{X}$ and $\mathrm{F}-\mathrm{CH}_{2}-\mathrm{CO}-\mathrm{X}$ systems, as well as selected conformers of an $\alpha, \beta$-difluoro- $\gamma$-amino-acid derivative that is potentially useful for the design of shape-controlled bioactive amino acids and peptides. We identify the most relevant exchange-correlation and/or electrostatic interaction terms contributing to the stability of the various conformers, and we show that IQA can assess the gauche/anti or trans/cis preferences in molecules with two or more rotatable bonds as well as to study the roles played by other concomitant effects (e.g., $\mathrm{CH} / \mathrm{OH} / \mathrm{NH} \cdots \mathrm{F}$ contacts). For the $\alpha, \beta$-difluoro- $\gamma$-amino acid derivatives, our theoretical analysis points out that the gauche/anti and trans/cis effects associated to fluorine bonds can be significantly attenuated by other specific intra-molecular contacts.
\end{abstract}




\section{Introduction}

In the last decades, fluorine has arisen as a remarkable element in numerous applications. Some of the most successful drugs on the market today include one or more fluorine atoms in their structures. Fluorine-containing molecules have made a significant contribution to the development of modern crop protection agrochemicals (herbicides, insecticides, and fungicides). ${ }^{2}, 3$ Fluorinated materials are widely used in liquid crystal displays ${ }^{4}$ and in photovoltaic solar cells. ${ }^{51}$ Specific fluorine substitution redefines the reactivity of nonfluorinated substrates and catalysts in terms of reaction yields, diastereo- or enantiomeric ratios, and mechanistic pathways. ${ }^{6,7}$ Similarly, fluorination modifies hydrophobicity, ring puckering and secondary structure propensity of amino-acids, and influences peptide/protein folding, stability and intermolecular interactions. ${ }^{8}$

The introduction of fluorine into a molecule can affect different physicochemical properties (i.e. hydrophobicity, reactivity, conformation, noncovalent interactions, etc.) due to electrostatic and stereoelectronic effects. Fluorine is a small atom that can replace hydrogen in organic molecules with a minimal steric impact, but with significant electronic consequences. It is the most electronegative element and, accordingly, the $\mathrm{C}-\mathrm{F}$ bond presents a high polarity (i.e. $\mathrm{C}^{\delta+}-\mathrm{F}^{\delta-}$ ) and a low polarizability. This results in a strong ionic character and a large dipole moment for the $\mathrm{C}-\mathrm{F}$ moiety that influence the chemical properties of adjacent groups. ${ }^{9}$ For example, $p K_{a}$ values of acetic acid and its successive $\alpha$ fluorinated derivatives change from $4.76\left(\mathrm{CH}_{3} \mathrm{COOH}\right)$ to $0.23\left(\mathrm{CF}_{3} \mathrm{COOH}\right)$ upon fluorine substitution. ${ }^{10}$ In addition, numerous examples show that stereoselective introduction of fluorine atoms within a molecule results in different conformational properties. ${ }^{11-15}$ On one hand, the $\mathrm{C}-\mathrm{F}$ bond tends to align antiparallel to adjacent $\mathrm{C}=\mathrm{O}$ bonds to confront the corresponding dipole moments, ${ }^{11}$ and to orient itself close to positively charged groups in order to maximize favorable charge/dipole interactions. ${ }^{16}$ On the other hand, the high electronegativity of fluorine determines the presence of a low energy $\sigma_{C-F}^{*}$ antibonding orbital that can accommodate electron density from stereoelectronically aligned lone pairs or adjacent $\sigma$ and $\pi$ bonds. Although this stereoelectronic effect (hyperconjugation) is energetically small, it has been traditionally invoked to explain the stabilization of certain molecular conformations. ${ }^{9}$

Traditionally, 1,2-difluoroethane has been considered the benchmark case for understanding the origin of the gauche structural preference (i.e. F-C-C-X angle around $60^{\circ}$ ). 
In previous computational works, ${ }^{17-19}$ the natural bond orbital (NBO) method ${ }^{20}$ ascribes the gauche preference in 1,2-difluoroethane to the $\sigma_{C H} \rightarrow \sigma_{C F}^{*}$ hyperconjugative interactions arising when adjacent $\mathrm{C}-\mathrm{H}$ and $\mathrm{C}-\mathrm{F}$ bonds align antiparallel. However, the ability of NBO to provide a right balance between Lewis (i.e. steric and electrostatic) and non-Lewis contributions has been questioned ${ }^{21}$ by the Energy Decomposition Analysis (EDA) approach, ${ }^{22}$ which similarly dismisses the central role of hyperconjugation in the conformational gauche effect. Thus, when EDA characterizes the interaction between two $\mathrm{CH}_{2} \mathrm{~F}$ radicals in the gauche/anti geometries of 1,2-difluoroethane, it turns out that equallyfavorable orbital and electrostatic interactions contribute to explain the gauche effect. ${ }^{23}$ Further support for the role of electrostatic interactions comes from an Interacting Quantum Atoms (IQA) analysis, in which the largest diatomic contribution to gauche stability is provided by the electrostatic interaction between carbon and fluorine atoms in positions $1,3 .{ }^{24}$ As a result, the authors conclude that the origin of the gauche effect in 1,2-difluoroethane is electrostatic rather than hyperconjugation.

Besides 1,2-difluoroethane, the conformational effects associated to the presence of fluorine atoms have been computationally analyzed in related compounds. For instance, DFT energy profiles connecting the gauche and anti conformers and atomic charges have been evaluated for a series of $16 \beta$-substituted $\alpha$-fluoroethanes $\left(F-\mathrm{CH}_{2} \mathrm{CH}_{2}-\mathrm{X}\right)$ in order to assess the influence of steric and electrostatic interactions, meanwhile NBO analysis are employed to disclose the role of hyperconjugation. ${ }^{18}$ These results point out that both electrostatic and hyperconjugative effects may contribute to conformer stability. In general, the role of hyperconjugation stabilizing the gauche structures increases with the electronegativity of the first atom in the $X$ moiety. Favorable electrostatic interactions between fluorine and electropositive atoms in the $X$ substituent also contribute to the gauche preference, with a remarkable gauche stabilization observed for positively charged groups like in 2fluoroethylammonium $\left(\mathrm{F}-\mathrm{CH}_{2} \mathrm{CH}_{2}-\mathrm{NH}_{3}{ }^{+}\right){ }^{16,18,25}$ It has been also found that $\mathrm{F}$ atoms adjacent to carbonyl groups stabilize the trans/gauche $\mathrm{F}-\mathrm{C}-\mathrm{C}=\mathrm{O}$ arrangements in the gas-phase, although this intrinsic preference can be reversed to the alternative cis form in polar solvents as suggested by DFT calculations coupled with a continuum solvent model. ${ }^{26-28}$ On the other hand, the NBO characterization of the 2-fluoroethanol $\left(\mathrm{F}-\mathrm{CH}_{2} \mathrm{CH}_{2}-\mathrm{OH}\right)$ conformers has explained the largest stability of a particular gauche structure by the presence of a favorable electrostatic $\mathrm{O}-\mathrm{H}^{\delta+\ldots} \delta \mathrm{F}$ interaction, which is termed as a non-classical hydrogen bond. ${ }^{29}$ In contrast, a very recent work interprets the ${ }^{4} \mathrm{JHF}$ couplings in $\alpha$-fluoro amides as a genuine $\mathrm{N}$ - 
$\mathrm{H} \cdots \mathrm{F}$ hydrogen bond. ${ }^{30}$ Finally, the anti/gauche conformers around the $\mathrm{F}-\mathrm{C}-\mathrm{C}-\mathrm{S}$ sequence in sulfur-containing systems have been subject to EDA considering the $\mathrm{FH}_{2} \mathrm{C}$. and $\cdot \mathrm{CH}_{2} \mathrm{SX}$ radicals as reference fragments. ${ }^{31}$ The electrostatic interaction favors preferentially the gauche conformer followed by the orbital interaction energy accounting for charge transfer effects. In the same study, ${ }^{31}$ NBO indicates that stereoelectronic effects work in favor of gauche conformers.

Other more complex systems have been also computationally examined to analyze the conformational effects associated to the introduction of fluorine atoms. DFT geometry optimizations followed by MP2 single point calculations have revealed that the conformational landscape of a series of 25 benzyl alcohols is significantly influenced by the presence of one or two fluorine atoms in the ortho position. ${ }^{32}$ Intramolecular interactions characterized in terms of Atoms in Molecules (AIM), Noncovalent Interactions (NCI) and NBO analyses, show that $\mathrm{OH} \cdots \mathrm{F}, \mathrm{CH} \cdots \mathrm{F}$ and/or $\mathrm{CH} \cdots \mathrm{O}$ intramolecular contacts contribute to the stabilization of the various conformations. ${ }^{32}$ On the other hand, the origin of the drastically different structure adopted by linear perfluoroalkanes and hydrocarbons (i.e helical vs zig-zag) has been inspected by means of $\mathrm{NBO} / \mathrm{NCl}$ analyses using DFT wave functions. The energetic preference for the helical structures increases monotonically with the chain length of the perfluorated alkane, what has been explained in terms of $\sigma_{C C} \rightarrow \sigma_{C F}^{*}$ hyperconjugative interactions, which are absent in the zigzag conformation. ${ }^{33}$ 
$\mathrm{F}_{2} \overbrace{1} \mathrm{~F}_{6}$

1: 1,2-difluoroethane

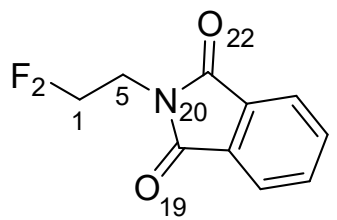

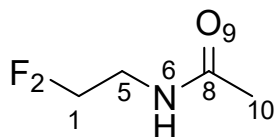

4: N-(2-fluoroethyl)acetamide

5: 2-(2-fluoroethyl)isoindoline-1,3-dione 6: 2-fluoroethan-1-aminium 7: 1-(2-fluoroethyl)pyridin-1-ium<smiles>O=C(I)C(F)I</smiles>

8: 1-fluoropropan-2-one<smiles>CCC(CF)C(F)F</smiles>

11: 1,2,3-trifluoropropane<smiles>[3H]OC(=O)C(F)I</smiles>

9: methyl-2-fluoroacetate<smiles>CNC(=O)C(F)C(F)F</smiles>

12: 2,3-difluoro- $N$-methyl propanamide

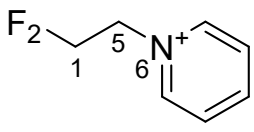

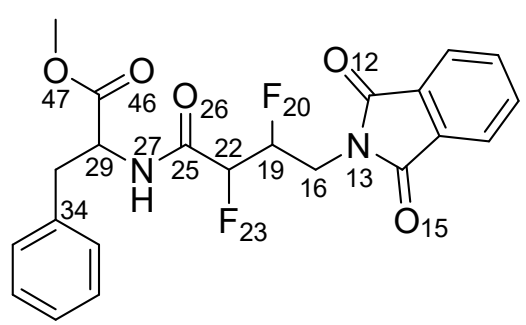

$14 a / 14 b$<smiles>CNC(=O)C(C)F</smiles>

10: 2-fluoro- $N$-methylacetamide<smiles>O=C1c2ccccc2C(=O)N1CC(F)CF</smiles>

13: 2-(2,3-difluoropropyl) isoindoline-1,3-dione

\section{Scheme 1}

From our review of the former computational investigations, it turns out that a variety of effects may be behind the conformational preferences observed in fluorinated systems. However, direct comparisons among the results obtained in these studies are largely hampered by the diversity of methodologies employed. Therefore, we pursue in this work to reexamine the conformational effects exerted by fluorine atoms using a common methodology based on the IQA energy partitioning augmented with pairwise dispersion energies. The dispersion-corrected IQA approach facilitates the decomposition of the global conformational energies into fragment contributions that can be further separated into various quantum mechanical and classical electrostatic terms. To better appreciate the conformational impact of these energy contributions, we will consider two classes of organic 
compounds. On one hand, we will evaluate intra and inter-atomic contributions to the relative energy of the gauche/anti conformers of fluoroethylene-derived systems (1-7 in Scheme 1), the trans/cis conformers in carbonyl containing systems (8-10), and the combined gauchegauche or gauche-trans preferences (11-13). On the other hand, we will characterize in detail the conformational properties of two diastereomers of an $\alpha, \beta$-difluoro- $\gamma$-amino-acid derivative (14a/14b in Scheme 1) that have been proposed to be particularly relevant for the design of shape-controlled bioactive amino acids and peptides, ${ }^{14}$ as they may link to other amino acids resulting in peptides with predictable conformational behavior. According to their crystal structures, the different stereochemistry at the $\alpha$ and $\beta$ carbons, $(R, R)$ for $14 \mathrm{a}$ and $(R, S)$ for 14b, results in a different backbone conformation (extended zig-zag in 14a and partially bent in 14b). Both structures exhibit the normally expected gauche orientation in the $\mathrm{F}_{23}-\mathrm{C}_{22}-\mathrm{C}_{19}$ $\mathrm{F}_{20}$ and $\mathrm{F}_{20}-\mathrm{C}_{19}-\mathrm{C}_{16}-\mathrm{N}_{13}$ bonds and the antiparallel alignment (trans-planar) of the $\mathrm{F}_{23}-\mathrm{C}_{22}-$ $\mathrm{C}_{25}=\mathrm{O}_{26}$ moiety. In fact, these three effects are supposed to determine the final conformation of the 14a and 14b molecules. All in all, we report theoretical results obtained for typical model systems prone to characterize specific conformational effects, but also for actual fluorinated compounds of synthetic and biochemical interest. The IQA methodology will allow us to treat them on the same basis, yielding thus a full energetic description of the various effects influencing their conformational preferences.

\section{Computational methods}

\section{IQA energy decomposition}

The IQA approach ${ }^{34}$ partitions the first- and second-order reduced density matrices into atomic regions such as the attraction basins $\left(\Omega_{\mathrm{A}}\right)$ that stem from the topological properties of the charge distribution $\rho(r)$ as commonly defined within the framework of the quantum theory of atoms in molecules (QTAIM). Two scalar fields derived from quantum mechanical (QM) wavefunctions are required to accomplish the IQA decomposition: the first order reduced density matrix $\rho_{1}\left(\mathbf{r}_{1}, \mathbf{r}_{1}{ }^{\prime}\right)$ and the pair density, $\rho_{2}\left(\mathbf{r}_{1}, \mathbf{r}_{2}\right)$. Then IQA decomposes the total energy of a molecular system in the gas-phase as

$$
E=\sum_{A} E_{n e t}^{A}+\sum_{A>B} E_{\mathrm{int}}^{A B}=\sum_{A}\left(T^{A}+V_{n e}^{A A}+V_{e e}^{A A}\right)+\sum_{A>B}\left(V_{n n}^{A B}+V_{n e}^{A B}+V_{n e}^{B A}+V_{e e}^{A B}\right)
$$


where $E_{\text {net }}^{A} \equiv E_{\text {net }}\left(\Omega_{A}\right)$ is the net electronic energy of atom A that includes the kinetic energy $T^{A}$ and the potential energy due to nuclei-electron (ne) attractions and electron-electron repulsions (ee) within $\Omega_{\mathrm{A}}$. The interaction energy $E_{\mathrm{int}}^{A B}=E_{\mathrm{int}}\left(\Omega_{A}, \Omega_{B}\right)$ between atoms $\mathrm{A}$ and $\mathrm{B}$ in the molecular system collects various potential energy terms ( $n n$, en, ne and ee). We stress that, according to the IQA terminology, an interaction energy is a diatomic contribution to the total energy of a molecule. In fact, the interaction energy $E_{\text {int }}^{A B}$ can be divided into classical and non-classical contributions, allowing thus to define a purely classical (electrostatic) component of the interaction energy, $E_{\mathrm{int}, \text { lass }}^{A B}=V_{n n}^{A B}+V_{n e}^{A B}+V_{n e}^{B A}+V_{e e, \text { Coul }}^{A B}$, along with a quantum (exchange-correlation) contribution such that $E_{\mathrm{int}}^{A B}=E_{\mathrm{int}, c l a s s}^{A B}+E_{\mathrm{int}, x c}^{A B}$. In this respect, we note also that the classical IQA components are distinguished only in the diatomic interaction terms $E_{\text {int }}^{A B}$, but not within the atomic net energies $E_{\text {net }}^{A}$.

By summing separately all the atomic and diatomic terms in equation (1), the total energy of a molecule is readily decomposed into net and interaction energy components ( $\left.E_{n e t}=\sum_{A} E_{n e t}^{A}, E_{\text {int,class }}=\sum_{A<B} \Delta E_{\text {int,class }}^{A B}, \ldots\right)$. By inserting the corresponding IQA energies for a given pair of gauche and anti conformers, the energy difference $E_{\text {gauche }}-E_{\text {anti }}$ can be written as

$$
\Delta E=E^{\text {gauche }}-E^{\text {anti }}=\Delta E_{\text {net }}+\Delta E_{\text {int,class }}+\Delta E_{\mathrm{int}, x c}
$$

\section{Dispersion corrected IQA}

In previous work, ${ }^{35}$ we have shown that IQA calculations can be complemented with the pairwise formulation of the third-generation dispersion (D3) correction for DFT and HF methods, ${ }^{36}$ which is a semiclassical potential inspired on the London formula for the dispersion attraction between two atoms $A$ and $B$ at large distance and that does not alter the underlying charge density. The correct asymptotic behavior of the $A-B$ dispersion energy can be reproduced using the Becke-Johnson $(\mathrm{BJ})^{37}$ rational damping function, resulting in the $\mathrm{D} 3(\mathrm{BJ})$ dispersion energy correction (termed simply as $\mathrm{D} 3$ in the manuscript) that can be readily combined with the IQA decomposition scheme. To this end, we simply add the dispersion interaction energies $E_{\mathrm{int}, d i s p}^{A B}$ to the rest of the IQA interaction energy terms such that the total D3-corrected IQA decomposition results, 


$$
E=\sum_{A} E_{\text {net }}^{A}+\sum_{A>B}\left(E_{\mathrm{int}, c l a s s}^{A B}+E_{\mathrm{int}, x c}^{A B}+E_{\mathrm{int}, d i s p}^{A B}\right)
$$

To keep the computational cost of the IQA calculations withtin reasonable bounds, all the IQA-D3 calculations are done with HF wavefunctions. In fact HF-D3 method provides a reasonable description of molecular structure and energetics, ${ }^{38,} 39$ and offers a straightforward physical partitioning because HF entirely lacks dispersion energy. To further assess the utility of the HF-D3 IQA decomposition, we briefly compare in the Supporting Information (Table S12) the HF-D3 IQA results on 1,2-difluoethane with those obtained with correlated methods (B3LYP-D3 and MP2).

\section{Interacting Quantum Fragments (IQF-D3)}

In principle, the grouping of the atomic IQA energy components into chemically-meaningful fragment contributions can be done using different notations and protocols depending on the particular problem at hand. For example, to characterize the conformational energy variation upon an internal rotation about a single bond, we will distinguish at least two molecular fragments, $P$ and $Q$, that are covalently linked $(P-Q)$ through the rotating bond. Such interacting quantum fragments (IQF) partitioning of a molecular system splits the total energy in intra-fragment and inter-fragment energy terms. The intra-fragment net energy ( $E_{n e t}^{P}$ and $E_{n e t}^{Q}$ ) collects the atomic net energies and the interaction energies among the atoms placed in the same fragment, whereas the inter-fragment term $\left(E_{\text {int }}^{P Q}\right)$ sums the classical and quantum interaction energies between atoms in different groups.

Using IQF-D3 quantities, the relative energy $\Delta E$ between the gauche and anti conformers around the $P-Q$ bond can be expressed as:

$$
\Delta E=\Delta E_{\text {net }}^{P}+\Delta E_{\text {net }}^{Q}+\Delta E_{\mathrm{int}, \text { lass }}^{P Q}+\Delta E_{\mathrm{int}, x c}^{P Q}+\Delta E_{\mathrm{int}, d i s p}^{P Q}
$$

where the relative IQF terms in the right hand are just the corresponding gauche/anti differences of the grouped terms $\left(e . g, \Delta E_{n e t}^{P}=E_{n e t}^{P, \text { gauche }}-E_{\text {net }}^{P, \text { anti }}\right)$ and the $\Delta E_{\mathrm{int}}^{P Q}$ term is split into the classical interaction energy $\Delta E_{\text {int,class }}^{P Q}$ and the purely $\mathrm{QM}$ correlation-exchange contribution $\Delta E_{\mathrm{int}, x c}^{P Q}$.

To further clarify the meaning of the IQF terms, it may be useful to briefly discuss the similarities and differences between IQF and the energy decomposition analysis (EDA).40, 41 
Assuming that EDA is applied to analyze the energy difference between two conformers of the $P-Q$ system with reference to separated (unrelaxed) $P$. and $Q$. radical fragments, $\Delta E$ would be the sum of three terms: a classical electrostatic interaction energy between $P$. and $Q$. ( $\left.V_{\text {essat }}\right)$, a Pauli repulsion term $\left(\Delta E_{\text {Pulli }}\right)$ that incorporates the kinetic and potential energy changes due to the antisymmetrization of the fragment wavefunctions, and an orbital relaxation energy $\left(\Delta E_{\text {orb }}\right)$ that arises from inter-fragment charge transfer and polarization effects. In general, the $\Delta E_{n e t}^{P}+\Delta E_{n e t}^{Q}+\Delta E_{\text {int, } x c}^{P Q}$ sum in IQF (termed as exchange-correlationrepulsion) resembles the $\Delta E_{\text {Palli }}+\Delta E_{\text {orb }}$ sum in EDA, while $\Delta E_{\text {int,class }}^{P Q}$ would correspond to $V_{\text {elsat }}$ although this identification is exact only in the limit of weakly interacting fragments for which $\Delta E_{\text {orb }}$ tends to zero. ${ }^{42}$ Nevertheless, the grouped EDA and IQF terms admit a similar interpretation on the basis of electrostatic and/or QM effects. However, the IQF balance between intra-fragment deformation $\left(\Delta E_{n e t}^{P}+\Delta E_{n e t}^{Q}\right)$ and inter-fragment exchange-correlation effects $\left(\Delta E_{\text {int } x c}^{P Q}\right)$ provides an alternative description of the $\mathrm{QM}$ effects embedded in the $\Delta E_{\text {Pauli }}+\Delta E_{\text {orb }}$ term without referring to separate fragments.

\section{Charge and Dipole electrostatic interactions}

In order to deepen the description of the IQF $\Delta E_{\text {int,lass }}^{P Q}$ term, the multipole expansion of the electrostatic potential may be useful for assessing the role of the charge distribution in the $P$ and $Q$ regions. In fact the QTAIM and IQA approaches allow us to express the Coulombic energy as a multicenter multipole expansion in a natural way..$^{43}$ It is well known that the electrostatic potential exerted by a set of point charges at a sufficiently distant point can be expanded in terms of multipole moments. In our study we focus on the first two contributions to the electrostatic potential, i.e., the total charge of a given basin $(q)$ and the dipole moment (d) emerged from its distribution. Hence, only the charge-charge, charge-dipole and dipoledipole interactions have been considered. Their respective analytical formulation are given in Eqs. (5)-(7) (in atomic units):

$$
\begin{aligned}
& E_{q q}=\frac{q^{P} q^{Q}}{R} \\
& E_{q d}=\frac{q^{P} \mu^{Q} \mathbf{R}}{R^{3}}
\end{aligned}
$$




$$
E_{d d}=\frac{R^{2} \boldsymbol{\mu}^{P} \boldsymbol{\mu}^{Q}-3\left(\boldsymbol{\mu}^{P} \mathbf{R}\right)\left(\boldsymbol{\mu}^{Q} \mathbf{R}\right)}{R^{5}}
$$

where the superscripts $P$ and $Q$ denote the two fragments considered, $\mathbf{R}$ represents the distance between their centers of mass, $q^{P} / q^{Q}$ are the total charge in $P / Q$ (i.e., $q^{P}=\sum_{A \in P} q^{A}$ ) and $\mu^{\mathrm{P}} / \mu^{\mathrm{Q}}$ are the dipole moment generated by the total charge distribution within $P / Q$.

Starting from the atomic dipoles arisen upon a particular 3D partitioning of the space, it is not, in general, straightforward to reconstruct fragment dipoles from atomic ones. The simple addition of the corresponding dipole components is not sufficient for the study of the charge-dipole or dipole-dipole fragment interactions. For neutral molecules but not for ions, $\mu$ is origin independent since any coordinate transformation $\mathbf{r}=\mathbf{r}{ }^{\prime}+\mathbf{R}_{0}$ gives

$$
\boldsymbol{\mu}=\int \mathbf{r} \rho(\mathbf{r}) d \mathbf{r}=\int \mathbf{r}^{\prime} \rho\left(\mathbf{r}^{\prime}+\mathbf{R}_{0}\right) d \mathbf{r}^{\prime}+\mathbf{R}_{0} \int \rho\left(\mathbf{r}^{\prime}+\mathbf{R}_{0}\right) d \mathbf{r}^{\prime}=\int \mathbf{r}^{\prime} \rho\left(\mathbf{r}^{\prime}+\mathbf{R}_{0}\right) d \mathbf{r}^{\prime}+\mathbf{R}_{0} q
$$

so that the charge-translation $\mathbf{R}_{0} q$ term vanishes for neutral molecules. To derive an atomic decomposition of $\mu$, the charge density $\rho$ can be partitioned into disjoint atomic regions with their own origin of coordinates at positions $\mathbf{R}_{0}^{A}$, and performing thus a transformation $\mathbf{r}=\mathbf{r}^{A}+\mathbf{R}_{0}^{A}$ in each basin. The total dipole is then expressed as

$$
\boldsymbol{\mu}=\sum_{A}\left(\int_{\Omega_{A}} \mathbf{r}^{A} \rho\left(\mathbf{r}^{A}+\mathbf{R}_{0}^{A}\right) d \mathbf{r}^{A}+\mathbf{R}_{0}^{A} \int_{\Omega_{A}} \rho\left(\mathbf{r}^{A}+\mathbf{R}_{0}^{A}\right) d \mathbf{r}^{A}\right)=\sum_{A}\left(\boldsymbol{\mu}_{i n t}^{A}+\mathbf{R}_{0}^{A} q^{A}\right)
$$

Eq. (9) shows that $\mu$ is the summation of atomic dipoles $\mu^{\mathrm{A}}$ that have two contributions: the intrinsic term $\boldsymbol{\mu}_{\text {int }}^{A}$ that comes from the integration of the dipole density function within the atomic basin plus the corresponding charge-translation term $\mathbf{R}_{0}^{A} q^{A}$. Fragment-based dipoles $\boldsymbol{\mu}^{P}$ can be likewise defined by considering both the intrinsic contributions and the net charges $q^{A}$ of the atomic basins that constitute fragment $P$ :

$$
\boldsymbol{\mu}^{P}=\sum_{A \in P} \boldsymbol{\mu}_{i n t}^{A}+\sum_{A \in P}\left(\mathbf{R}_{0}^{A}-\mathbf{R}_{0}^{P}\right) q^{A}
$$

The reconstructed dipoles $\boldsymbol{\mu}^{P}$ depend on the origin of coordinates which, in this work, are selected as the center of mass of fragment $P\left(\mathbf{R}_{0}^{P}\right)$. We note that, in the case of neutral molecules, the dependency of the $\mu^{P}$ values on the origin of coordinates is small. We also 
emphasize that the total electrostatic interaction energy among the various atoms/fragments in a given molecule computed from the multipolar expansion is always origin-independent.

\section{QM calculations}

Initial coordinates for the small and medium-sized models were generated using the UCSF Chimera program. ${ }^{44}$ Starting from 1,2-difluoroethane in the gauche conformation, all the $\mathrm{CH}_{2} \mathrm{~F}-\mathrm{CH}_{2} \mathrm{X}$ systems were built in the gauche conformation by replacing the second fluorine atom by the appropriate $X$ group. Then the initial geometries of the corresponding anti conformers were obtained by adjusting the F-C-C-X angle to $180^{\circ}$. Similarly, we built the initial trans/cis conformers for the $\mathrm{CH}_{2} \mathrm{~F}-\mathrm{COX}$ systems.

All the gauche/anti and trans/cis structures of the small models were fully optimized in the gas-phase with no symmetry constraints at the HF-D3/cc-pVTZ and the RI-MP2/cc-pVTZ levels of theory. These calculations were done with the ORCA 4.0.1 package. ${ }^{45}$ The HFD3/cc-pVTZ energy minimizations were carried out with the D3 dispersion energy and gradient corrections choosing the Becke-Johnson (BJ) damping function. ${ }^{36,37}$ The resolutionof-the-identity (RI) approximation was activated for the MP2 calculations using the appropriate auxiliary basis set. To further estimate electron correlation effects on the conformational energies, we employed the domain-based Local Pair Natural Orbital (DLPNO) coupled cluster method ${ }^{45,46}$ as implemented in the ORCA 4.0.1 package. More specifically, we performed DLPNO-CCSD(T)/aug-cc-pVTZ single point calculations on the MP2/cc-pVTZ geometries using tight thresholds to control the DLPNO approximations. The DLPNO$\operatorname{CCSD}(T)$ method exhibits near linear scaling at the cost of introducing small deviations from the canonical coupled cluster methods. For tight DLPNO thresholds, the typical errors with respect to canonical results are below $0.25 \mathrm{kcal} / \mathrm{mol} .{ }^{47}$

For 1,2,3-trifluoropropane and the related $\mathrm{CH}_{2} \mathrm{~F}-\mathrm{CHF}-\mathrm{CH}_{2} \mathrm{X}$ system with an isoindolederivative as the $X$ substituent, initial coordinates were generated by setting the $\mathrm{F}-\mathrm{C}-\mathrm{C}-\mathrm{F}$ and F-C-C-X angles to $\pm 60^{\circ}$ and/or $180^{\circ}$, resulting in nine structures accordingly named $\mathrm{g}^{+} / \mathrm{g}^{+}$, $g^{+} / g^{-}, g^{+} / a n t i$, etc. In the case of the $\mathrm{CH}_{2} \mathrm{~F}-\mathrm{CHF}-\mathrm{CONHCH}_{3}$ molecule, the $\mathrm{F}-\mathrm{C}-\mathrm{C}=\mathrm{O}$ angle takes the values $180^{\circ}$ for the trans and $0^{\circ}$ for the cis conformers, which combined with the three options available for the F-C-C-F torsion result in six initial structures termed $g+/$ trans, g+/cis, g-/trans, etc. As in the smaller $\mathrm{CH}_{2} \mathrm{~F}-\mathrm{CH}_{2} \mathrm{X}$ systems, initial geometries were fully optimized in the gas-phase at the HF-D3/cc-pVTZ and the RI-MP2/cc-pVTZ levels of theory 
followed by single point DLPNO-CCSD(T)/aug-cc-pVTZ calculations on the MP2/cc-pVTZ geometries.

For the 14a and $14 \mathrm{~b}$ compounds, automatic conformational analyses were performed using the multiconformer generator MS-DOCK program. ${ }^{48}$ Starting from the corresponding crystal structures,${ }^{14}$ we used the antechamber program to optimize the initial geometries and to assign AM1-BCC atomic charges and SYBYL atom types. ${ }^{49}$ Subsequently, 50 different conformers were generated for $\mathbf{1 4 a}$ and $\mathbf{1 4 b}$ using the MS-DOCK software and the resulting structures were optimized using the MMFF94 force field. During conformer generation, a filter based on the root mean squared deviation of the Cartesian coordinates and the energy was applied to eliminate similar structures. The $14 \mathbf{a}$ and $\mathbf{1 4 b}$ different conformers were then optimized at the HF-D3/cc-pVDZ level of theory using the ORCA 4.0.1 program. ${ }^{45}$ After inspection of the structure and energy of the minimized structures, only 20 different conformers remained for $\mathbf{1 4 a}$ and $\mathbf{1 4 b}$. These were reoptimized at the HF-D3/cc-pVTZ level of theory using ORCA both in the gas phase and in chloroform using the SMD continuum solvent model. ${ }^{50}$ For the five more stable conformers of $14 a$ and $\mathbf{1 4 b}$, selected NMR vicinal proton-fluorine coupling constants ${ }^{3} \mathrm{~J}_{\mathrm{H}, \mathrm{F}}$ were estimated using a seven-parameter Karplustype relation. ${ }^{51}$ The equation correlates a ${ }^{3} \mathrm{~J}, \mathrm{~F}$ value with the corresponding $\mathrm{H}-\mathrm{C}-\mathrm{C}-\mathrm{F}$ torsion angle and it also includes correction terms for substituent electronegativity a well as for $\mathrm{H}-\mathrm{C}$ $\mathrm{C}$ and $\mathrm{F}-\mathrm{C}-\mathrm{C}$ bonds angles. The ${ }^{3} \mathrm{~J}$, F values were computed using the HF geometries optimized in chloroform, whereas the electronegativity values for the $\mathrm{C} \alpha-\mathrm{C} \beta$ substituents (1.4 for fluorine, 0.9 for carbon and 0.0 for hydrogen) were taken from reference 51 .

\section{Promolden calculations}

The IQA decomposition of molecular energies at the HF-D3/cc-pVTZ level was performed with a modular version of the PROMOLDEN program ${ }^{52}$ that is being developed in our laboratory. As previously noticed, the pairwise dispersion energies computed with the DFTD3 program ${ }^{53}$ are combined with the various IQA terms $\left(E_{\text {net }}\left(\Omega_{A}\right), E_{\text {int }}\left(\Omega_{A}, \Omega_{B}\right), \ldots\right)$ to formulate the IQA-D3 energy decomposition of the corresponding HF-D3 energies (i.e., three-body dipole-dipole-dipole dispersion energy is not included). The IQA quantities are numerically integrated over the atomic basins $\Omega_{\mathrm{A}}$, which constitute finite and irregular integration domains, using very large angular and radial grids in atomic spherical quadratures. We adopted integration settings that represent a compromise choice between computational cost and accuracy for small and medium-sized molecules. Thus, a $\beta$-sphere around each atom 
was considered (i.e., a sphere completely contained inside the atomic basin), with a radius equal to $60 \%$ the distance of its nucleus to the closest bond critical point in the electron density. High-quality Lebedev angular grids were used with 5810 and 974 points outside and within the $\beta$-spheres of heavy atoms, respectively, (3890 and 590 points for hydrogen atoms). Euler-McLaurin radial quadratures were employed with 512 and 384 radial points outside and inside the $\beta$-spheres of heavy atoms, respectively (384 and 256 points for $H$ ). The largest value of the radial coordinate in the integrations was 15.0 au for heavy atoms $(10.0$ au for $\mathrm{H}$ atoms). Maximum angular moments, $\lambda_{\max }$, of 10 and 6 were assigned to the Laplace and bipolar expansions of the $1 / r_{12}$ operator outside and within the $\beta$-spheres.

Most of the IQA calculations reported in this work were computed with the conventional $\mathcal{O}\left(N^{4}\right)$ algorithm implemented in PROMOLDEN that employs the $N$ occupied canonical molecular orbitals (MO) to expand the first and second-order density matrices. For the $14 \mathbf{a} / \mathbf{1 4 b}$ compounds that have 51 atoms, the $\mathcal{O}\left(N^{4}\right)$ algorithm is exceedingly expensive and, therefore, we used a variant that uses localized MOs and employs the multipolar approach ${ }^{54}$ for computing selected interatomic exchange-correlation (xc) energies. The LMOs were computed with the Pipek-Mezey algorithm ${ }^{55}$ as implemented in the ORCA 4.0.1 package. For each atomic basin $\Omega_{A}$, a subset of LMOs $\left\{\phi_{i}^{L M O}\right\}_{A}$ is then built by requiring that their diagonal contribution to the atomic overlap matrix $\left(\int_{\Omega_{A}}\left|\phi_{i}^{L M O}\right|^{2} d \tau\right)$ is greater than $10^{-6} \mathrm{au}$. The calculation of the IQA $E_{n e t}\left(\Omega_{A}\right)$ terms are done using the corresponding subset $\left\{\phi_{i}^{L M O}\right\}_{A}$ for each basin. For the calculation of the diatomic $E_{\text {int }}\left(\Omega_{A}, \Omega_{B}\right)$ terms, the LMO sets of the pair of basins are combined as the union $\left\{\phi_{i}^{L M O}\right\}_{A} \cup\left\{\phi_{j}^{L M O}\right\}_{B}$ or intersection $\left\{\phi_{i}^{L M O}\right\}_{A} \cap\left\{\phi_{j}^{L M O}\right\}_{B}$ in order to integrate the Coulombic or exchange-correlation interactions, respectively. The multipolar $x C$ approximation at high order of the angular momentum series $(L=10)$ is activated for $1-n(n>4)$ intramolecular interactions provided that the interatomic $R_{A B}$ distance is greater than 5.0 au. For $R_{A B}>17 \mathrm{au}$, the $E_{\text {int }}^{x c}\left(\Omega_{A}, \Omega_{B}\right)$ values are neglected. The goodness of these additional approximations was tested by comparing the results of conventional (MO-based) and LMObased IQA calculations on the conformers of 2,3-difluoro- $N$-methylpropanamide and 2-(2,3difluoropropyl)isoindoline-1,3-dione (12 and 13 in Scheme 1). The IQA error defined as $\left|E-E^{I Q A}\right|$, where $E$ is the total energy obtained from the QM calculations and $E^{I Q A}$ is the total 
energy reconstructed from the IQA terms, had average values of $0.5 \mathrm{kcal} / \mathrm{mol}$ (12) and 0.9 $\mathrm{kcal} / \mathrm{mol}$ (13) for the MO-based IQA calculations. The corresponding values for the LMO/multipolar-based IQA calculations were similar: $0.5 \mathrm{kcal} / \mathrm{mol}$ (12) and $1.2 \mathrm{kcal} / \mathrm{mol}$ (13). Hence, the additional numerical error due to the LMO and multipolar approximations is expected to be small without compromising the conclusions of the IQA analysis.

\section{Results}

As above mentioned, 1,2-difluoroethane is usually considered as the reference system to analyze the gauche/anti conformational preference associated to adjacent fluorine atoms. For this reason, initial models for the gauche (F-C-C-F 60 ) and anti (F-C-C-F 180 $)$ conformers of 1,2-difluoroethane were optimized in the gas phase at the HF-D3/cc-pVTZ and MP2/cc-pVTZ levels of theory. Both geometry optimizations provided very similar structures, with the largest differences in the geometries located in the C-F bond lengths (see Figure 1). Relative energies were further reevaluated by means of DLPNO-CCSD(T)/aug-cc-pVTZ single point calculations performed on the MP2 geometries (see Figure 1), the gauche conformer being $0.7 \mathrm{kcal} / \mathrm{mol}$ more stable than the antione in agreement with previous results (unless otherwise noticed $\Delta E$ values in the text correspond to DLPNO-CCSD(T) data).

Clearly, the magnitude of the gauche effect in 1,2-difluoroethane seems too scarce to allow the design of molecules with a frozen conformation. Hence, it is interesting to examine how the gauche preference can be modulated in other systems. Actually, replacing one of the fluorine atoms in 1,2-difluoroethane for other functional groups could drive away the energy of the gauche and anti conformers, increasing the impact of a single C-F bond in selecting a particular conformation. To further clarify this point, we optimized the gauche and anti conformers for a number of $\beta$-substituted $\alpha$-fluoroethanes (see 1-7 in Scheme1 and Figure 1). Among the neutral molecules in Figure 1, the largest gauche/anti conformational preference is achieved for acetamide with $1.6 \mathrm{kcal} / \mathrm{mol}$ favoring the gauche conformation. A more pronounced gauche preference is induced by a positive charge adjacent to the fluorine atom. Thus, gauche structures with either ammonium or pyridinium groups are 6.6 and 4.2 $\mathrm{kcal} / \mathrm{mol}$, respectively, more stable than the corresponding anti ones. 
Figure 1. Ball-and-stick representation of the gauche/anti conformers optimized for different $\mathrm{CH}_{2} \mathrm{~F}-\mathrm{CH}_{2}-\mathrm{X}$ systems. The C-F and C-C bond distances $(\AA)$, the F-C-C-X dihedral angle $\left(^{\circ}\right)$, and selected intramolecular distances $(\AA)$ measured in the HF-D3/cc-pVTZ optimized structures (RI-MP2/cc-pVTZ in parentheses) are shown. Energy differences $\left(E_{\text {gauche }}-E_{\text {anti }}\right)$ in $\mathrm{kcal} / \mathrm{mol}$ computed at the HF-D3/cc-pVTZ, RI-MP2/cc-pVTZ (in parentheses) and DLPNO-CCSD(T)/aug-cc-pVTZ [in brackets] levels of theory are also included.

1: 1,2-difluoroethane

$\Delta E=-0.28(-0.76)[-0.75]$

2: 2-fluoroethyl-acetate

$\Delta E=-0.52(-0.85)[-0.84]$

gauche

anti

gauche

3: 3-fluoropropanal

$\Delta E=-0.91 \quad(-0.65)[-0.68]$

4: $N$-(2-fluoroethyl)acetamide

$\Delta E=-1.50(-1.59)[-1.65]$

gauche

5: 2-(2-fluoroethyl)isoindoline-1,3-dione anti

gauche

$\Delta E=-0.28(-0.46)[-0.42]$ anti

gauche
6: 2-fluoroethan-1-ammonium
$\Delta E=-6.53(-6.63)[-6.56]$
7: 1-(2-fluoroethyl)pyridin-1-ium
$\Delta E=-4.16(-4.30)[-4.21]$ 
When a carbonyl group is placed adjacent to a C-F bond as in $\alpha$-fluoroamides, the trans-planar arrangement of the $\mathrm{F}-\mathrm{C}-\mathrm{C}=\mathrm{O}$ moiety is energetically favored over the cis one. This is observed in Figure 2 for a ketone (8) and for an amide (10). In contrast, we computed an almost negligible cis/trans conformational preference for the ester (9).

Figure 2. Ball-and-stick representation of the cis/trans conformers optimized for different $\mathrm{CH}_{2} \mathrm{~F}-\mathrm{CO}-\mathrm{X}$ systems. The C-F and C-C distances $(\AA)$, the F-C-C=O dihedral angle $\left(^{\circ}\right)$ and selected intramolecular distances $(\AA)$ measured in the HF-D3/cc-pVTZ optimized structures (RI-MP2/cc-pVTZ in parentheses) are shown. Energy differences $\left(E_{\text {trans }}-E_{c i s}\right)$ in $\mathrm{kcal} / \mathrm{mol}$ computed at the HF-D3/cc-pVTZ, RI-MP2/cc-pVTZ (in parentheses) and DLPNO-CCSD(T)/aug-cc-pVTZ (in squared brackets) levels of theory are also included.

$$
\text { 8: 1-fluoropropan-2-one } \quad \Delta E=-2.79(-2.06)[-2.09]
$$

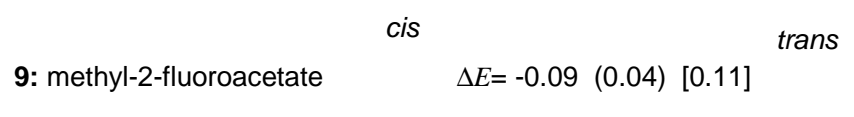

cis

trans

10: 2-fluoro- $N$-methylacetamide $\quad \Delta E=5.80(5.81)$ [5.64]

To analyze the origin of these energetic preferences, we carried out the IQA partitioning of the conformational energy differences at the HF-D3/cc-pVTZ level. In this respect, we note that relative energies in Figures 1 and 2 confirm that the HF-D3 $\Delta E$ values remain reasonably close to the $\operatorname{DLPNO}-\operatorname{CCSD}(\mathrm{T})$ ones, the mean unsigned difference between them being $0.2 \mathrm{kcal} / \mathrm{mol}$. Hence, we believe that this favorable comparison validates the use of the HF-D3 method in the subsequent IQA analyses. 
As described in Methods, IQA ${ }^{56}$ decomposes molecular energy differences into a collection of atomic and interatomic terms. For 1,2-difluoroethane, the combination of the IQA quantities including the small dispersion contribution, results in an IQA reconstructed $\Delta E=E_{\text {gauche }}-E_{\text {ant }}$ energy difference of $-0.5 \mathrm{kcal} / \mathrm{mol}$, which is close to the HF-D3/cc-pVTZ reference value $(-0.3$ $\mathrm{kcal} / \mathrm{mol})$. This discrepancy is due to the numerical errors accumulated during the calculation of the IQA quantities. As the individual IQA terms are typically one or two orders of magnitude larger than $\Delta E$, their expected relative error would be small.

Inspection of the IQA energies shows that the largest differences between the gauche and anti conformers arise in the electrostatic interactions (see Table S2). Thus, the gauche conformation is largely favored by the $\Delta E_{\text {int,class }}$ term corresponding to the $\mathrm{C}_{1} \cdots \mathrm{F}_{6}(-8.0$ $\mathrm{kcal} / \mathrm{mol})$ and $\mathrm{F}_{2} \cdots \mathrm{C}_{5}(-7.9 \mathrm{kcal} / \mathrm{mol})$ electrostatic contacts. However, this gauche stabilization is widely compensated by the repulsive $F_{2} \cdots F_{6}(12.1 \mathrm{kcal} / \mathrm{mol})$ and $C_{1} \cdots C_{5}(6.9 \mathrm{kcal} / \mathrm{mol})$ electrostatic terms. Moreover, the overall gauche preference is also affected by other atomic and diatomic IQA terms, as the $\mathrm{C}_{1} \cdots \mathrm{C}_{5}$ exchange-correlation interaction $(-3.2 \mathrm{kcal} / \mathrm{mol})$, the $F_{2}, F_{6}$, and $C_{1}$ electronic distortions ( $\Delta E_{n e t}=1.1,1.0$ and $1.0 \mathrm{kcal} / \mathrm{mol}$, respectively), etc. By summing over all the atomic and diatomic contributions (see Table S1), it arises that both the classical electrostatic $\left(\Delta E_{\text {int,class }}=-2.3 \mathrm{kcal} / \mathrm{mol}\right)$ and the exchange-correlation $\left(\Delta E_{\text {int }, x c}=-1.9\right.$ $\mathrm{kcal} / \mathrm{mol}$ ) interactions favor the gauche conformation in 1,2-difluoroethane.

By considering the 1,2-difluoroethane molecule as formed by two interacting quantum fragments (IQF), the gauche/anti conformational energy difference can be split into intrafragment deformation terms and electrostatic and quantum-mechanical inter-fragment contributions. Due to the symmetric character of the two $\mathrm{CH}_{2} \mathrm{~F}$ groups, there is no net charge transfer between them so that $\Delta E_{\text {net }}^{P}$ and $\Delta E_{\text {net }}^{Q}$ collect the energetic impact of electronic reorganization within $P$ and $Q$ and of a minimal steric hindrance. The computed net energy differences for each $\mathrm{CH}_{2} \mathrm{~F}$ group are 0.6 and $0.4 \mathrm{kcal} / \mathrm{mol}$ (these distinct values are again due to IQA numerical errors), but the overall deformation $(1.0 \mathrm{kcal} / \mathrm{mol})$ favoring the anti orientation is clearly smaller than the inter-fragment energy changes (see Table 1). Thus, the largest contribution to the stabilization of the gauche conformer comes from the non-classical exchange-correlation interaction $(-3.5 \mathrm{kcal} / \mathrm{mol})$ between the $\mathrm{CH}_{2} \mathrm{~F}$ groups. In contrast, classical electrostatic effects clearly stabilize the anti conformer $(2.1 \mathrm{kcal} / \mathrm{mol})$, which is usually explained in terms of the repulsion arising from the nearly aligned $\mathrm{C}$ - $\mathrm{F}$ dipole moments 
in the gauche orientation (i.e., F-C-C-F dihedral $\sim 60^{\circ}$ ). This interpretation seems partly correct because, according to our analyses, the dipole-dipole interaction accounts for $55 \%$ of the difference in the $\mathrm{CH}_{2} \mathrm{~F} \cdots \mathrm{CH}_{2} \mathrm{~F}$ electrostatic interaction between the gauche/anti conformers so that higher-order multipolar contributions would also be important. Anyway, it is the $\Delta E_{\text {int }, x c}^{P Q}+\Delta E_{\text {int,class }}^{P Q}$ sum $(-1.4 \mathrm{kcal} / \mathrm{mol})$ that mainly controls the stability of the gauche conformer and, therefore, the IQF energy decomposition for 1,2-difluoroethane resembles those in previous proposals about the combined role of hyperconjugation and electrostatics to explain the gauche effect. ${ }^{23}$ Within the context of IQF, we propose then to measure the gauche stabilization in 1,2-difluoroethane and related systems as $\Delta E_{\text {int }, x c}^{P Q}+\Delta E_{\text {int, class }}^{P Q}$.

Probably, either the IQA or the IQF analysis of the gauche/anti 1,2-difluoroethane may be adequate and useful. Nonetheless, chemists usually explain molecular properties in terms of the functional groups or the characteristic moieties that build up a molecule. For large systems, grouping the atomic terms into fragments simplifies the interpretation of the IQA data because the IQF terms smooth out the correlated fluctuations of the atomic quantities within a given group. Therefore, we decided to focus on the IQF results for the rest of the systems studied in this work, although the underlying atomic partitioning will also be used to better characterize some relevant inter- or intra-fragment contributions.

Table 1. IQF energy components at the HF-D3/cc-pVTZ level for the energy difference (kcal/mol) between the two confomers (gauche/anti or cis/trans) analyzed for a series of small systems related to 1,2-difluoroethane. Two-fragment partitioning scheme $\left(P=\mathrm{CH}_{2} \mathrm{~F}\right.$ and $\left.Q=\mathrm{CH}_{2} \mathrm{X}\right)$ is assumed for the different systems.

\begin{tabular}{|c|c|c|c|c|c|c|c|c|c|c|}
\hline$\Delta E=E_{\text {gauche }}-E_{\text {anti }}$ & $\Delta q^{P}$ & $\Delta E_{n e t}^{P}$ & $\Delta E_{\text {net }}^{Q}$ & $\Delta E_{\text {int,disp }}$ & $\Delta E_{\text {int }, x c}$ & $\Delta E_{\text {int,class }}$ & $\Delta E_{\text {int,class }, q q}$ & $\Delta E_{\text {int,class, } q d}$ & $\Delta E_{\text {int,class,dd }}$ & $\Delta E_{I Q A}$ \\
\hline 1: 1,2-difluoroethane & 0.00 & 0.6 & 0.4 & -0.0 & -3.5 & 2.1 & 0.0 & 0.0 & 1.1 & -0.5 \\
\hline 2: 2-fluoroethyl-acetate & 0.00 & 0.7 & 0.5 & -0.0 & -3.1 & 1.4 & 0.0 & $0.0 \quad 0.0$ & -0.7 & -0.5 \\
\hline 3: 3-fluoropropanal & -0.01 & 0.0 & 1.4 & -0.0 & -1.1 & -1.5 & -0.1 & $-0.4 \quad 0.0$ & -0.4 & -1.2 \\
\hline 4: $N$-(2-fluoroethyl)acetamide & -0.02 & -0.6 & 2.1 & -0.2 & -2.1 & -1.4 & -0.2 & $\begin{array}{ll}-0.6 & 0.1\end{array}$ & -1.0 & -2.1 \\
\hline 5: 2-(2-fluoroethyl)isoindoline-1,3-dione & 0.00 & 2.0 & 1.5 & -0.3 & -3.6 & -0.1 & 0.0 & $-0.0-0.0$ & 0.1 & -0.5 \\
\hline 6: 2-fluoroethxan-1-aminium & -0.01 & 1.6 & 4.7 & -0.6 & -6.9 & -6.0 & 2.4 & $1.3-3.5$ & -4.6 & -7.3 \\
\hline 7: 1-(2-fluoroethyl)pyridin-1-ium & -0.01 & 1.0 & 3.5 & -0.3 & -5.5 & -3.7 & 0.9 & $0.2-6.1$ & -0.3 & -5.1 \\
\hline$\Delta E=E_{\text {trans }}-E_{\text {cis }}$ & $\Delta q^{P}$ & $\Delta E_{n e t}^{P}$ & $\Delta E_{\text {net }}^{Q}$ & $\Delta E_{\text {int,disp }}$ & $\Delta E_{\text {int }, x c}$ & $\Delta E_{\text {int,class }}$ & $\Delta E_{\text {int,class }, q q}$ & $\Delta E_{\text {int,class, } q d}$ & $\Delta E_{\text {int,class,dd }}$ & $\Delta E_{I Q A}$ \\
\hline 8: 1-fluoropropan-2-one & 0.01 & 0.1 & 0.6 & 0.0 & 1.5 & -4.9 & 0.0 & $\begin{array}{ll}-0.1 & 0.1\end{array}$ & -2.9 & -2.8 \\
\hline 9: methyl-2-fluoroacetate & 0.00 & 0.6 & 0.5 & 0.0 & 0.3 & -1.4 & 0.0 & 0.70 .2 & -2.8 & -0.1 \\
\hline 10: 2-fluoro-N-methylacetamide & -0.02 & 2.1 & 2.7 & -0.2 & -4.1 & -6.3 & 0.0 & 0.20 .1 & -5.2 & -5.8 \\
\hline
\end{tabular}


Substituent effects on the gauche/anti $\mathrm{CH}_{2} \mathrm{~F}-\mathrm{CH}_{2} \mathrm{X}$ conformers: Competition between IQF exchange-correlation and electrostatic interactions

For the $\mathrm{CH}_{2} \mathrm{~F}-\mathrm{CH}_{2} \mathrm{X}$ molecules, the gauche conformer is energetically favored over the anti one (see Figure 1). In all the $\mathrm{CH}_{2} \mathrm{~F}-\mathrm{CH}_{2} \mathrm{X}$ molecules, the charge variation at the $\mathrm{CH}_{2} \mathrm{~F} / \mathrm{CH}_{2} \mathrm{X}$ moieties upon the anti $\rightarrow$ gauche rearrangement is null or minimal $(\sim 0.01 \mathrm{e})$ as computed from the corresponding QTAIM charges.

Regarding 1,2-difluoroethane as the reference compound, we observe that replacing the second fluorine atom by an acetate group ( 2 in Scheme 1 ) results in very small changes in the IQF terms. Again the gauche conformer is stabilized by the exchange correlation interaction $\left(\Delta E_{\text {int, } x c}=-3.1 \mathrm{kcal} / \mathrm{mol}\right)$. Electrostatics favors the anti geometry $\left(\Delta E_{\text {int,class }}=1.4\right.$ $\mathrm{kcal} / \mathrm{mol})$, but such effect does not arise from favorable $\mu-\mu$ interactions $\left(\Delta E_{\text {int,class, } d d}=-0.7\right.$ $\mathrm{kcal} / \mathrm{mol})$. This is due to the internal geometry of the acetate group, in which the dipole moments along the $\mathrm{C}=\mathrm{O} / \mathrm{C}-\mathrm{O}$ bonds combine in a total fragment dipole moment $(\sim 1.9 \mathrm{D})$ that is nearly orthogonal to the $\mathrm{CH}_{2} \mathrm{~F}$ dipole ( 2.2 D) in the anti conformer.

Introduction of an aldehyde (3) or an amide (4) group leads to classical and exchangecorrelation inter-fragment interactions that stabilize the gauche conformer, being partially compensated by the unfavorable net energy change of the $\mathrm{CH}_{2} \mathrm{X}$ fragment (see Table 1). There are, however, some differences between the aldehyde and the amide groups. The largest inter-atomic electrostatic interaction involves the fluorine atom in the two systems, but the $\mathrm{F}_{2} \cdots \mathrm{C}_{8}$ interaction in the aldehyde 3 stabilizes the gauche structure $(-15.9 \mathrm{kcal} / \mathrm{mol})$, meanwhile the equivalent $\mathrm{F}_{2} \cdots \mathrm{N}_{6}$ contact stabilizes the anti conformation in $4(27.9 \mathrm{kcal} / \mathrm{mol})$. If we compare the electrostatic $\mathrm{F}_{2} \cdots \mathrm{N}_{6}$ interaction in the amide with $\mathrm{F}_{2} \cdots \mathrm{O}_{6}$ in $2(20.8 \mathrm{kcal} / \mathrm{mol})$ and $F_{2} \cdots F_{6}$ in $1(12.1 \mathrm{kcal} / \mathrm{mol})$, it arises an inverse relationship between the electronegativity of the $\mathrm{X}$ atom in the F-C-C-X sequence and the penalty for the gauche orientation due to the $\mathrm{F} \cdots \mathrm{X} \Delta E_{\text {int,class }}$ electrostatic term.

In contrast with the 1-4 systems, the small gauche stabilization computed for the isoindole derivative 5 arises from a large and favorable exchange-correlation term (-3.6 $\mathrm{kcal} / \mathrm{mol}$ ), mainly ascribed to the $\mathrm{H}_{3} \cdots \mathrm{O}_{19}$ and $\mathrm{F}_{2} \cdots \mathrm{N}_{20}$ interactions (see Table S2). But this favorable $\Delta E_{\text {int }, x c}$ term is cancelled by the intra-fragment net energies $(2.0$ and $1.5 \mathrm{kcal} / \mathrm{mol}$ ). The change in the inter-fragment electrostatic interaction is almost negligible $(-0.1 \mathrm{kcal} / \mathrm{mol})$ due to the cancelation of large inter-atomic electrostatic interactions between fluorine and the heteroatoms in the isoindole moiety. 
A cationic group adjacent to a C-F bond significantly stabilizes the gauche conformation, what is commonly explained in terms electrostatics. In effect, the $\Delta E_{\text {int,class }}$ terms in the gauche-anti energy difference in $\mathrm{CH}_{2} \mathrm{~F}-\mathrm{CH}_{2} \mathrm{X}^{+}$become quite important: -6.0 and -3.7 $\mathrm{kcal} / \mathrm{mol}$ for the ammonium (6) and pyridinium (7) groups. These two cationic substituents have different size and charge distribution, what is reflected in their $\mu^{Q}$ dipole moments, $\sim 2.5$ $\mathrm{D}$ and $\sim 0.2 / 0.5 \mathrm{D}$ for the ammonium and pyridinium $\mathrm{CH}_{2} \mathrm{X}^{+}$groups, respectively. The global positive charge is delocalized between the $P$ and $Q$ fragments (e.g., $q^{P}=0.12$ and $q^{Q}=0.88$ in the gauche 6 conformer) and these $q^{P} / q^{Q}$ charges repel more strongly in the gauche orientation. Thus, the charge-dipole and dipole-dipole fragment interactions explain the electrostatic preference for the gauche location of the ammonium group (see Table 1), while the rather small $\mu^{Q}$ value of pyridinum implies that only the interaction between the bulkier pyridinium charge and the $\mathrm{CH}_{2} \mathrm{~F}$ dipole makes a relevant contribution to the gauche stability $(-6.1 \mathrm{kcal} / \mathrm{mol})$. As in the $\mathrm{CH}_{2} \mathrm{~F}-\mathrm{CH}_{2} \mathrm{X}$ neutral systems, the change in the fragment net energies stabilizes the anti orientation in the cationic systems albeit with a more pronounced influence (e.g., $\Delta E_{\text {net }}^{Q}=4.7$ and $3.5 \mathrm{kcal} / \mathrm{mol}$ for 6 and 7 ). However, the IQF data reveals that the exchange-correlation inter-fragment interactions reinforce the stability of the gauche orientation (see Table 1) and compensate the deformation energies. More particularly, in the gauche 6 structure, a short $\mathrm{F} \cdots \mathrm{H}-\mathrm{N}$ contact rationalizes well the significant $\Delta E_{i n t, x c}^{P Q}$ value of $6.9 \mathrm{kcal} / \mathrm{mol}$ (see Table S2 and Figure 1).

\section{IQF description of trans/cis conformers in $\mathrm{CH}_{2} \mathrm{~F}-(\mathrm{C}=\mathrm{O}) \mathrm{X}$ : Electrostatic control}

When a carbonyl group is placed adjacent to a fluorine atom, electrostatic effects are assumed to stabilize the trans-planar orientation of the $\mathrm{C}=\mathrm{O}$ and $\mathrm{C}-\mathrm{F}$ groups over the cis one (see Figure 2). Our IQF calculations on the $\alpha$-fluoroketone (8), $\alpha$-fluoroester (9), and $\alpha$ fluoroamide (10) molecules confirm that the electrostatic interaction between the $\mathrm{CH}_{2} \mathrm{~F}$ and $(\mathrm{C}=\mathrm{O}) \mathrm{X}$ moieties is the key element that explains the larger stability of the $\mathrm{C}-\mathrm{F} / \mathrm{C}=\mathrm{O}$ trans arrangement $\left(\Delta E_{\text {int,class }}=-4.9,-1.4\right.$ and $-6.2 \mathrm{kcal} / \mathrm{mol}$ for $\mathbf{8}, 9$ and 10; see Table 1). Furthermore, this electrostatic effect is mainly associated to the dipole-dipole interaction term in the three cases, although contributions from higher multipoles seem also relevant for the $\alpha$-fluoroketone 8. The exchange-correlation term also contributes to the stability of the trans fluoroamide $(-4.1 \mathrm{kcal} / \mathrm{mol})$, whereas it favors the cis orientation in the fluoroketone $(1.5$ 
$\mathrm{kcal} / \mathrm{mol}$ ) and has little effect on the $\alpha$-fluoroester. These $\Delta E_{i n t, \mathrm{xc}}$ contributions mainly result from the $\mathrm{F}_{2} \cdots \mathrm{O}_{6}(4.5 \mathrm{kcal} / \mathrm{mol}) / \mathrm{F}_{2} \cdots \mathrm{C}_{7}(-3.1)$ interactions in the cis/trans $\alpha$-fluoroketone 8 , and the $\mathrm{F}_{2} \cdots \mathrm{N}_{7}(-5.3) / \mathrm{F}_{2} \cdots \mathrm{O}_{6}(4.7) / \mathrm{F}_{2} \cdots \mathrm{H}_{8}(-3.0)$ contacts in the $\alpha$-fluoroamide 10. Nonetheless, the favorable $\mathrm{F} \cdots \mathrm{H}-\mathrm{N}$ polar contact in $\mathbf{1 0}$ is nearly compensated by significant fragment distortion (see $\Delta E_{n e t}^{P}$ and $\Delta E_{\text {net }}^{Q}$ in Table 1) so that the electrostatic $\Delta E_{\text {int,class }}$ is the major stabilizing energy contribution to the trans preference between the vicinal $\mathrm{C}-\mathrm{F}$ and $\mathrm{C}=\mathrm{O}$ groups.

-CF-CF-CF- motif: IQA discrimination among gauche effects, 1,3 dipole-dipole and 1,3 $\mathrm{CH} \cdots \mathrm{F}$ contacts

For large molecules bearing various fluorine atoms as well as other functional groups, several fluorine-associated effects may control their conformational preferences. For instance, the preferred conformations in polyfluorinated alkanes have been assumed to arise from maximizing gauche interactions between vicinal fluorine atoms and, simultaneously, minimizing unfavorable dipole-dipole interactions between 1,3-difluoro motifs. ${ }^{9,13}$ To further analyze these effects, we optimized the nine conformers of 1,2,3-trifluoropropane (11) generated by the gauche $g+\left(\sim 60^{\circ}\right)$, the gauche $g-\left(\sim-60^{\circ}\right)$ or the anti $\left(\sim 180^{\circ}\right)$ orientations of the two consecutive F-C-C-F torsions. All the conformers remain within a narrow energy range of $\sim 3 \mathrm{kcal} / \mathrm{mol}$. In addition, some of the structures correspond to equally-stable conformational enantiomers (pair $g+/ g+$ and $g-/ g-$, pair $g+/ a n t i$ and anti/g-, and pair $g$-/anti and anti/g+) and, accordingly, only the first conformer within each pair was considered for the IQA analysis. Since the relative energies for the 1-7 compounds are given as $\Delta E=E_{\text {gauche }}-E_{\text {anti }}$, the relative energies for $\mathrm{CH}_{2} \mathrm{~F}-\mathrm{CHF}-\mathrm{CH}_{2} \mathrm{~F}$ given in the text and in Figure 3 are similarly expressed as $\Delta E=E_{g+/ g+}-E_{i}$ where $i$ stands for any other conformer of 11. Hence, a negative $\Delta E$ value means that conformer $i$ is less stable than the $g+/ g+$ one. 
Figure 3. Ball-and-stick representation of the different conformers obtained for 1,2,3-trifluoropropane (11 in Scheme 1). F-C-C-F dihedral angles $\left(^{\circ}\right)$ and selected interatomic distances $(\AA)$ measured in the HF-D3/cc-pVTZ optimized structures (RI-MP2/cc-pVTZ in parentheses) are shown. Energy differences $\left(E_{g_{+} / g_{+}}-E_{i}\right)$ in $\mathrm{kcal} / \mathrm{mol}$ computed at the HF-D3/cc-pVTZ, RI-MP2/cc-pVTZ (in parentheses) and DLPNO-CCSD(T)/aug-cc-pVTZ [in brackets] levels of theory are also included.

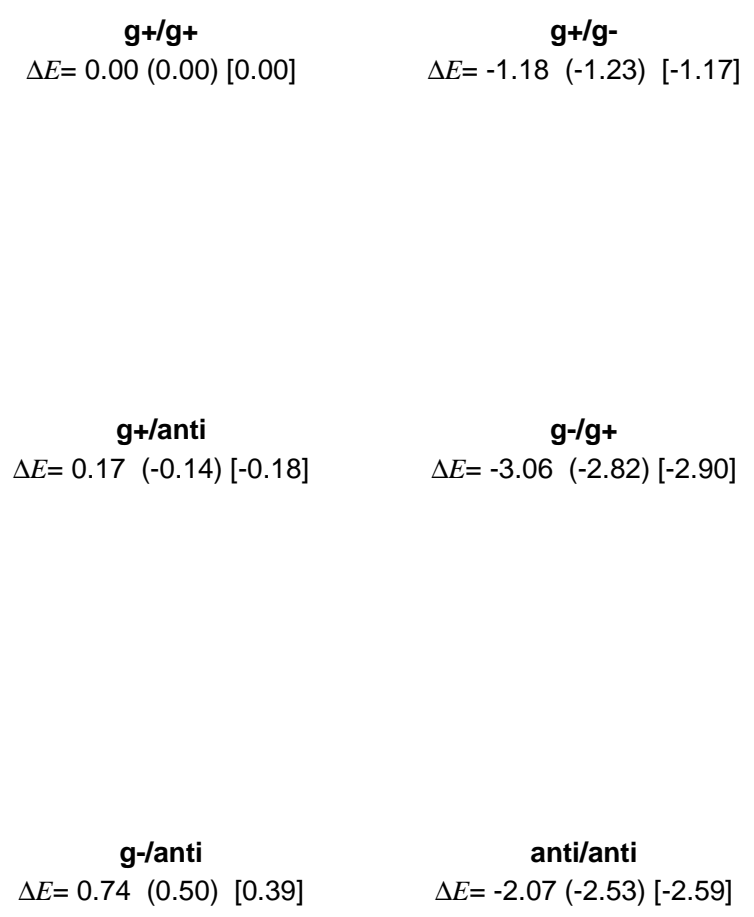

Table 2 collects the IQF energy decomposition of the relative energies with respect to the reference $g+/ g$ + structure considering three molecular fragments (i.e., $P=\mathrm{CH}_{2} \mathrm{~F}-, Q=-\mathrm{CHF}$ , and $R=-\mathrm{CH}_{2} \mathrm{~F}$ ). In line with previous proposals, the parallel alignment of the $\mathrm{C}_{1}-\mathrm{F}_{2}$ and $\mathrm{C}_{8}$ F9 bonds in the less stable $g$ - $/ g+$ and antilanti conformers is associated to unfavorable electrostatic interactions between the corresponding $\mathrm{CH}_{2} \mathrm{~F}$ groups $\left(\Delta E_{\mathrm{int}, \text { class }}^{P \cdots}\right.$ of -2.4 and -2.1 $\mathrm{kcal} / \mathrm{mol}$, respectively), which are mainly ascribed to dipole-dipole repulsion ( $\Delta E_{\mathrm{int}, \text { lass }, d d}^{p, R}$ of -2.0 and $-2.1 \mathrm{kcal} / \mathrm{mol}$ for $g-/ g+$ and anti/anti). Hence, it seems reasonable to describe them as "1,3-dipole repulsions" in consonance with former suggestions. ${ }^{9}$ 
Table 2. IQF relative energy components (in $\mathrm{kcal} / \mathrm{mol}$ ) at the HF-D3/cc-pVTZ level for the energetically different conformers of 1,2,3-trifluoropropane (11). A three-fragment partitioning scheme $\left(P=\mathrm{CH}_{2} \mathrm{~F}, Q=\mathrm{CHF}\right.$, and $\left.R=\mathrm{CH}_{2} \mathrm{~F}\right)$ is assumed for the different structures. Energy differences are computed as $E_{g_{+} / g_{+}}-E_{i}$, so that a negative value means further stabilization of $g+/ g+$.

\begin{tabular}{|c|c|c|c|c|c|c|c|c|c|c|c|c|c|c|}
\hline & \multirow[t]{2}{*}{$\Delta E_{\text {net }}^{P}$} & \multirow[t]{2}{*}{$\Delta E_{\text {net }}^{Q}$} & \multirow[t]{2}{*}{$\Delta E_{n e t}^{R}$} & \multicolumn{3}{|c|}{$\Delta E_{\text {int,disp }}$} & \multicolumn{3}{|c|}{$\Delta E_{\text {int }, x c}$} & \multicolumn{3}{|c|}{$\Delta E_{\text {int,class }}$} & \multirow[t]{2}{*}{$\Delta E_{I Q A}$} & \multirow[t]{2}{*}{$\Delta E_{H F-D 3}$} \\
\hline & & & & $P \cdots Q$ & $P \cdots R$ & $Q \cdots R$ & $P \cdots Q$ & $P \cdots R$ & $Q \cdots R$ & $P \cdots Q$ & $P \cdots R$ & $Q \cdots R$ & & \\
\hline$\Delta E=E_{g+/ g+}-E_{g+/ g-}$ & 2.4 & -1.9 & 0.6 & -0.0 & -0.1 & 0.0 & 0.6 & -1.4 & 0.1 & -0.3 & -0.6 & -0.9 & -1.5 & -1.2 \\
\hline$\Delta E=E_{g+/ g+}-E_{g+/ a n t i}$ & 0.7 & -0.7 & 0.8 & -0.0 & -0.0 & 0.0 & 1.1, & -0.2 & -2.3 & -0.4 & -0.1 & 1.5 & 0.4 & 0.2 \\
\hline$\Delta E=E_{g+/ g+}-E_{g-/ g+}$ & 0.6 & 0.1 & -1.4 & 0.0 & -0.3 & 0.0 & 0.1 & -0.7 & -0.2 & 0.8 & -2.4 & 0.2 & -3.2 & -3.1 \\
\hline$\Delta E=E_{g+/ g+}-E_{g-\text {-anti }}$ & 0.4 & 1.3 & -1.7 & -0.0 & 0.0 & 0.0 & 0.5 & 1.0 & -2.7 & 0.2 & 0.1, & 1.6 & 0.8 & 0.7 \\
\hline$\Delta E=E_{g+/ g+}-E_{\text {anti/anti }}$ & 1.7 & 0.3 & -0.5 & -0.0 & -0.3 & 0.0 & -1.8 & -1.1 & -2.1 & 2.1, & -2.1 & 1.6 & -2.1 & -2.1 \\
\hline
\end{tabular}

The gauche effect is also expected to play a key role in determining the conformational landscape of 1,2,3-trifluoropropane. However, the missing gauche effects between vicinal fluorine atoms do not penalize the antilanti structure as compared to the $g-/ g+$ one (i.e., antilanti is $1.0 \mathrm{kcal} / \mathrm{mol}$ more stable than $g-/ g+$ at HF-D3/cc-pVTZ). The lack of a net gauche effect in the antilanti structure is revealed by IQF because the $\Delta E_{\text {int,xc }}$ terms between consecutive fragments favoring the gauche arrangement $(-1.8$ for $P \cdots Q$ and $-2.1 \mathrm{kcal} / \mathrm{mol}$ for $Q \cdots R)$ are nearly compensated by electrostatic interactions $\left(\Delta E_{\text {int,class }}=2.1\right.$ and $1.6 \mathrm{kcal} / \mathrm{mol}$ for $P \cdots Q$ and $Q \cdots R)$ favoring the anti conformation. Other differences between the $g-/ g+$ and antilanti structures arise in the intra-fragment net energies, that disfavor the $g-/ g+$ structure ( $\left.\Delta E_{\mathrm{net}}^{R}=-1.4 \mathrm{kcal} / \mathrm{mol}\right)$ though they stabilize the antilanti one $\left(\Delta E_{\mathrm{net}}^{P}=1.7 \mathrm{kcal} / \mathrm{mol}\right)$.

The $g+/ a n t i$ and $g$-/anti conformers, with only one gauche arrangement, are nearly isoenergetic $(g+/ a n t i)$ or slightly more stable $(g$-/anti) than the reference $g+/ g+$ conformer presenting two gauche effects (see Figure 3). IQF confirms that the gauche effect at $\mathrm{F}_{6-} \mathrm{C}_{5-}$ $\mathrm{C}_{8}-\mathrm{F}_{9}$ favors $g+/ g+$ over $g+/$ anti and $g$-/anti, because the exchange-correlation $\Delta E_{\mathrm{int}, x c}^{Q \cdots R}$ term (2.3 and $-2.7 \mathrm{kcal} / \mathrm{mol}$ for $g+/$ anti and $g$-/anti, respectively) is greater in absolute value than the electrostatic $\Delta E_{\text {int,class }}^{Q \cdots R}$ term $(1.5 \mathrm{kcal} / \mathrm{mol}$ for $g+/$ anti and $1.6 \mathrm{kcal} / \mathrm{mol}$ for $g$-/anti $)$. The same comparison involving the $\Delta E_{\mathrm{int}, x c}^{P \cdots Q}$ and $\Delta E_{\mathrm{int}, \text { class }}^{P \cdots Q}$ terms suggest that the $g+/$ anti conformer may exhibit a reinforced gauche preference in the $\mathrm{F}_{2}-\mathrm{C}_{1}-\mathrm{C}_{5}-\mathrm{F}_{6}$ angle.

Inspection of the optimized geometries in Figure 3 suggests that $\mathrm{C}-\mathrm{H} \cdots \mathrm{F}$ contacts can contribute to the conformational energies. As expected, the formation/loss of the $\mathrm{C}-\mathrm{H} \cdots \mathrm{F}$ contacts can be traced back to modifications in either the deformation or the interaction IQA components involving the $\mathrm{C}-\mathrm{H} \cdots \mathrm{F}$ atoms (Table S4). The best fingerprint of the $\mathrm{C}-\mathrm{H} \cdots \mathrm{F}$ 
contact is provided by the exchange-correlation $\Delta E_{\mathrm{in}, x c}^{A B}$ energy between the $\mathrm{F}$ and $\mathrm{H}$ atoms because it scores among the largest $\Delta E_{\mathrm{in}, x c}^{A B}$ terms and correlates well with the IQF exchangecorrelation energy. For example, the reference $g+/ g+$ structure has an $\mathrm{H}_{4} \cdots \mathrm{F}_{9}$ contact (2.57 $\AA$ ) which is replaced by a similar $\mathrm{H}_{3} \cdots \mathrm{F}_{9}$ interaction in the $g+/ a n t i$ conformer $(2.53 \AA)$, and this change is linked to significant interatomic $\Delta E_{\text {int, } x c}^{A B}$ contributions $\left(-1.6 \mathrm{kcal} / \mathrm{mol}\right.$ for $\mathrm{H}_{4} \cdots \mathrm{F}_{9}$ and $1.9 \mathrm{kcal} / \mathrm{mol}$ for $\mathrm{H}_{3} \cdots \mathrm{F}_{9}$ in Table S4). In the case of $g$-/anti, the $\mathrm{H}_{4} \cdots \mathrm{F}_{9}$ interaction in $\mathrm{g}+\mathrm{g}+\mathrm{g}$ is preserved and one additional $\mathrm{F}_{2} \cdots \mathrm{H}_{10}$ contact $(2.49 \AA)$ is formed that results in a favorable interatomic $\mathrm{F}_{2} \cdots \mathrm{H}_{10} \Delta E_{\text {int,xc }}$ contribution (2.3 kcal/mol in Table S4). Furthermore, the lack of $1,3 \mathrm{C}-\mathrm{H} \cdots \mathrm{F}$ contacts in the $g+/ g$ - structure (e.g. $\Delta E_{\text {int } x c}=-1.7 \mathrm{kcal} / \mathrm{mol}$ for $\mathrm{H}_{4} \cdots \mathrm{F}_{9}$ ) can be invoked to explain its lower stability $\left(1.2 \mathrm{kcal} / \mathrm{mol}\right.$ with respect to $\left.g_{+} / g+\right)$ in spite of maintaining two gauche effects. Hence, we conclude that the conformational properties of 1,2,3trifluoropropane are more significantly influenced by through-space exchange-correlation interactions between fluorine and hydrogen atoms than the gauche effect between vicinal fluorine atoms, and that the IQA/IQF signature of these contacts is conveniently described in terms of the exchange-correlation interaction energies.

\section{IQF analysis of fragments from an $\alpha, \beta$-difluoro- $\gamma$-amino-acid}

To help ascertain all the effects determining the conformational properties of the $\alpha, \beta$-difluoro$\gamma$-amino-acids 14a/14b containing the -CHF-CHF- motif, we first examined compound 12 formally derived from 11 by replacing one fluorine atom by a polar amide group (see Scheme 1). Either a gauche or an anti orientation in the adjacent $\mathrm{C}-\mathrm{F}$ bonds and a cis or a trans arrangement for the vicinal $\mathrm{C}-\mathrm{F}$ and $\mathrm{C}=\mathrm{O}$ bonds can appear in 12 (see Figure 4). Only five conformers were located on the HF and MP2 potential energy surfaces ( $g$-/cis turned out to be unstable). Their relative energies given in Figure 4 are expressed as $\Delta E=E_{g+\text { trans }}-E_{i}$ where $i$ stands for any other conformer of 12.

The positioning of the $-(\mathrm{C}=\mathrm{O}) \mathrm{NHCH}_{3}$ group in $\mathbf{1 2}$ results in a strong conformational selection in favor of the $\mathrm{g}+/$ trans or $\mathrm{g}$-/trans conformers, which is unequivocally interpreted by the three-fragment $\left(P=\mathrm{CH}_{2} \mathrm{~F}, Q=\mathrm{CHF}\right.$, and $\left.R=\mathrm{CONHCH}_{3}\right)$ IQF partitioning. As already observed in 10, the cis alignment in the adjacent $\mathrm{C}_{5}-\mathrm{F}_{6}$ and $\mathrm{C}_{8}=\mathrm{O}_{9}$ bonds in 12 is clearly destabilized by the classical electrostatic interaction between fragments $Q$ and $R$. The 
corresponding $\Delta E_{\text {int,lass }}^{Q \cdots R}$ values are -7.5 and -8.4 for the $g+/ c i s$ and anti/cis conformers, the exchange-correlation $\Delta E_{\mathrm{int}, \mathrm{xc}}^{Q \cdots R}$ being also unfavorable (see Table 3). Some differences appear in the $P \cdots Q$ and $P \cdots R$ interaction terms that contribute to the relative stability of the $g+/ c i s$ and anti/cis conformers. Thus, the lack of gauche effect between vicinal $\mathrm{C}-\mathrm{F}$ bonds makes anti/cis less stable than $g+/$ trans $\left(\right.$ e.g., $\left.\Delta E_{\text {int,xc }}^{P \cdots Q}+\Delta E_{\text {int,class }}^{P \cdots Q}=-2.8+1.5=-1.3 \mathrm{kcal} / \mathrm{mol}\right)$. On the other hand, the terminal $P \cdots R$ interaction through a $\mathrm{F}_{2} \cdots \mathrm{H}_{11}-\mathrm{N}_{10}$ contact favors anti/cis $\left(\Delta E_{\mathrm{int}, \mathrm{xc}}^{P \cdots \cdot R}=3.0\right.$ $\mathrm{kcal} / \mathrm{mol}$ and $\left.\Delta E_{\text {int,class }}^{P \ldots R}=3.4 \mathrm{kcal} / \mathrm{mol}\right)$ over $g+/ c$ is $\left(\Delta E_{\text {int,xc }}^{P \ldots R}=0.4 \mathrm{kcal} / \mathrm{mol}\right.$ and $\Delta E_{\text {int,class }}^{P \cdots R}=-2.0$ $\mathrm{kcal} / \mathrm{mol})$. With respect to the electrostatic terms, the partial alignment of the $\mathrm{C}_{1}-\mathrm{F}_{2}$ and amide dipole moments contributes to further destabilize $g+/$ cis (the $P \cdots Q$ dipole-dipole interaction amounts to $-1.7 \mathrm{kcal} / \mathrm{mol}$ ).

Figure 4. Ball-and-stick representation of the five conformers optimized for 2,3-difluoro- $N$-methylpropanamide (12 in Scheme 1). F-C-C-F and F-C-C-C=O dihedral angles $\left(^{\circ}\right)$ and selected interatomic distances $(\AA)$ measured in the HF-D3/cc-pVTZ optimized structures (RI-MP2/cc-pVTZ in parentheses) are included. Energy differences $\left(E_{\mathrm{g}+\text { /trans }}-E_{i}\right)$ in $\mathrm{kcal} / \mathrm{mol}$ computed at the HF-D3/cc-pVTZ, RI-MP2/cc-pVTZ (in parentheses) and DLPNO$\operatorname{CCSD}(T) /$ aug-cc-pVTZ [in brackets] levels of theory are also included. 
The magnitude of the relative energy of the anti/trans conformer of $12(4.2 \mathrm{kcal} / \mathrm{mol})$ as compared to the $g+/$ trans structure is difficult to explain only in terms of the small gauche effect estimated as $\Delta E_{\mathrm{in}, \mathrm{xc}}^{P \cdots}+\Delta E_{\mathrm{int}, \mathrm{class}}^{P \cdots}=-3.4+2.9=-0.5 \mathrm{kcal} / \mathrm{mol}$. In contrast, the terminal $P \cdots R$ interactions, $\Delta E_{\mathrm{int}, \mathrm{xc}}^{P \cdots R}=-1.6 \mathrm{kcal} / \mathrm{mol}$ and $\Delta E_{\mathrm{int}, \mathrm{class}}^{P \cdots R}=-2.6 \mathrm{kcal} / \mathrm{mol}$, make a more significant contribution to the destabilization of anti/trans. These terms can be associated with the loss of the $\mathrm{H}_{3} \cdots \mathrm{O}_{9}$ contact $\left(\Delta E_{\mathrm{int}, \mathrm{xc}}^{H_{3} \cdots O_{9}}=-2.1 \mathrm{kcal} / \mathrm{mol}\right.$ in Table S5) when comparing $g+/$ trans with anti/trans, while the electrostatic contribution can be connected to the partial alignment of the $P$ and $R$ dipole moments in the anti/trans structure (the $P \cdots R$ dipole-dipole interaction amounts to $-2.6 \mathrm{kcal} / \mathrm{mol})$. Thus, we see again that either 1,3 dipole-dipole interactions or $\mathrm{O} / \mathrm{F} \ldots \mathrm{H}$ contacts can be more determining than the gauche effect between adjacent C-F groups.

Table 3. IQF energy components at the HF-D3/cc-pVTZ level for the energy difference $(\mathrm{kcal} / \mathrm{mol})$ among the five conformers optimized for 2,3-difluoro- $N$-methylpropanamide (12). A three-fragment partitioning scheme $\left(P=\mathrm{CH}_{2} \mathrm{~F}-, Q=-\mathrm{CHF}-\right.$, and $\left.R=-\mathrm{CONHCH}_{3}\right)$ is assumed for the different conformers. Energy differences are computed as $E_{g+\text { trans }}-E_{i}$, so that a negative value means further stabilization of $g+/$ trans.

\begin{tabular}{|c|c|c|c|c|c|c|c|c|c|c|c|c|c|c|}
\hline & \multirow[t]{2}{*}{$\Delta E_{n e t}^{P}$} & \multirow[t]{2}{*}{$\Delta E_{n e t}^{Q}$} & \multirow[t]{2}{*}{$\Delta E_{n e t}^{R}$} & \multicolumn{3}{|c|}{$\Delta E_{\text {int, disp }}$} & \multicolumn{3}{|c|}{$\Delta E_{\text {int }, x c}$} & \multicolumn{3}{|c|}{$\Delta E_{\text {int,class }}$} & \multirow[t]{2}{*}{$\Delta E_{I Q A}$} & \multirow[t]{2}{*}{$\Delta E_{H F-D 3}$} \\
\hline & & & & $P \cdots Q$ & $P \cdots R$ & $Q \cdots R$ & $P \cdots Q$ & $P \cdots R$ & $Q \cdots R$ & $P \cdots Q$ & $P \cdots R$ & $Q \cdots R$ & & \\
\hline$\Delta E=E_{g+/ \text { trans }}-E_{g-\text { trans }}$ & -0.8 & -1.7 & 0.6 & 0.0 & -0.1 & 0.0 & -0.1 & -0.4 & 1.3 & 0.0 & 0.4 & -0.0 & -0.7 & -0.2 \\
\hline$\Delta E=E_{g+t \text { trans }}-E_{\text {antittrans }}$ & 0.1 & -1.7 & 0.7 & -0.0 & -0.4 & 0.0 & -3.4 & -1.6 & 1.7 & 2.9, & -2.6 & -0.1 & -4.3 & -4.2 \\
\hline$\Delta E=E_{g+\text { trans }}-E_{g+/ \text { cis }}$ & 1.1 & 1.7 & 2.0 & -0.0 & 0.3 & -0.4 & -0.5 & 0.4 & -4.8 & 0.4 & -2.0 & -7.5 & -9.3 & -8.0 \\
\hline$\Delta E=E_{g+\text { trrans }}-E_{\text {anticis }}$ & 0.3 & 0.3 & 0.1 & 0.0 & 0.6 & -0.4 & -2.8 & 3.0, & -3.9 & 1.5, & 3.4, & -8.4 & -6.3 & -5.5 \\
\hline
\end{tabular}

In contrast with the case of compound 12, the positioning of the bulky isoindole-1,3dione group $-(\mathrm{C}=\mathrm{O}) \mathrm{NHCH}_{3}$ group in 13 has a minor conformational influence. For this reason, its analysis is reported in the Supplementary Material (Figure S1 and Tables S6-S7).

\section{Conformational analysis and QM calculations on the $14 a$ and $14 b$ dipeptides}

The QM and IQA calculations on the model compounds 1-13 characterize several conformational effects (gauche/anti, cis/trans, through space $\mathrm{F} \cdots \mathrm{H}$, dispersion attractions, etc.) than can act simultaneously in the $\mathbf{1 4 a / 1 4 b}$ dipeptides. To better assess these and other effects, we investigated first whether or not additional conformations to those observed in the 14a/14b crystal structures could be accessible. Thus, we performed an automated conformational search followed by HF-D3/cc-pVTZ geometry optimizations of the resulting 
conformers in the gas-phase and in the solvent continuum (see Tables S8-S9 and Figures S2-S3).

Most remarkably, inspection of the optimized geometries and relative energies of the 14a and 14b conformers reveals that the X-ray conformations (labelled as 14a-conf1 and 14b-conf1) are indeed among the most stable ones in the gas-phase and in the chloroform solution. In addition, we found that the structures presenting the expected $\mathrm{F}_{23}-\mathrm{C}_{22}-\mathrm{C}_{19}-\mathrm{F}_{20}$ and $\mathrm{F}_{20}-\mathrm{C}_{19}-\mathrm{C}_{16}-\mathrm{N}_{13}$ gauche orientation and the trans $\mathrm{F}_{23}-\mathrm{C}_{22}-\mathrm{C}_{25}=\mathrm{O}_{26}$ arrangement (see Figure 5 for atom numbering) are, in general, energetically favored as expected by common assumptions. There are, however, low energy conformers, especially for $\mathbf{1 4 a}$, that do not present the expected gauche orientation around the fluorine atoms (see for instance 14aconf4 and 14a-conf5). Moreover, it turns out that the conformer 14a-conf6 is not drastically penalized in the gas-phase despite presenting a cis $\mathrm{F}_{23}-\mathrm{C}_{22}-\mathrm{C}_{25}=\mathrm{O}_{26}$ alignment and an almost anti orientation for the $\mathrm{F}_{23}-\mathrm{C}_{22}-\mathrm{C}_{19}-\mathrm{F}_{20}$ and $\mathrm{F}_{20}-\mathrm{C}_{19}-\mathrm{C}_{16}-\mathrm{N}_{13}$ bonds.

The results of our conformational search can be addressed with NMR experimental data. Thus, the NMR spectra of $14 a$ and $14 b$ have been recorded at $300 \mathrm{~K}$ in deuterated chloroform solution and their coupling constants determined by simulation/iteration sequences. ${ }^{14}$ We also estimated ${ }^{3} J_{H F}$ coupling constants around the $\mathrm{FC}_{22}-\mathrm{C}_{19} \mathrm{~F}$ bond using a Karplus-type relation ${ }^{51}$ for the five most stable conformers of $14 a$ and $14 \mathbf{b}$, and the corresponding values were Boltzmann-averaged at $300 \mathrm{~K}$ according to the HF-D3/cc-pVTZ relative energies in chloroform (Table S10). For 14a, the favorable comparison between the computed and experimental values validates the results of our conformational analysis (the computational averages for ${ }^{3} J_{F H}\left(\mathrm{~F}_{23} \mathrm{H}_{21}\right)$ and ${ }^{3} J_{H F}\left(\mathrm{H}_{24} \mathrm{~F}_{20}\right)$ are 27.4 and $28.2 \mathrm{~Hz}$ compared with the experimental ones of 27.0 and $28.9 \mathrm{~Hz}$ ). It is interesting to note that some conformational variability of 14 a in chloroform solution can be expected because the $\Delta E$ difference between the two most stable conformers is not large $(1.0 \mathrm{kcal} / \mathrm{mol})$. For $14 \mathbf{b}$, the agreement between the estimated coupling constants and the experimental ones is only moderate (the calculated averages for ${ }^{3} J_{F H}$ and ${ }^{3} J_{H F}$ are 26.1 and $13.0 \mathrm{~Hz}$ while the experimental values are 23.0 and $21.4 \mathrm{~Hz}$ ). However, the computed ${ }^{3} J_{F H}$ and ${ }^{3} J_{H F}$ values for 14b-conf1 (30.8 and 8.1) and 14b-conf2 (6.6 and 31.7) would approach to the experimentally-derived ones (23.0 and 21.4) upon averaging, provided that these two conformers become nearly isoenergetic. Although the calculated $\Delta E$ value between 14bconf1 and $14 \mathrm{~b}$-conf 2 is already small $(0.7 \mathrm{kcal} / \mathrm{mol})$, it is likely that method $/$ basis-set 
improvements in the QM level of theory and/or a better description of thermal and solvation effects would be required to increase the agreement with experimental data.

\section{IQF assessment of the conformational effects acting upon 14a}

Our QM calculations suggest that intramolecular factors largely determine the conformational preferences of $\mathbf{1 4 a / 1 4 b}$ as their low-energy conformers in the gas-phase are quite close to their crystallographic structures. Moreover, the intrinsic stability of the two diastereomers is similar and we expect that the same local effects influence their conformational energies. Taking also into account the large computational cost of the IQA calculations on these systems, we focused on the IQA analysis of the relative stability of selected 14a conformers. Thus, we performed IQA calculations on 14a-conf1 and 14a-conf2, which are two different gauche forms ( $g+$ and $g$-, respectively) that interconvert into each other through a $\sim 120^{\circ}$ rotation about the $\mathrm{F}_{23} \mathrm{C}_{22}-\mathrm{C}_{19} \mathrm{~F}_{20}$ bond. We also selected conformer 14a-conf5, which shows an anti conformation at $\mathrm{F}_{23}-\mathrm{C}_{22}-\mathrm{C}_{19}-\mathrm{F}_{20}$, and conformer 14a-conf6, which is a helical-type compact structure presenting all anti $\left(\mathrm{F}_{23}-\mathrm{C}_{22}-\mathrm{C}_{19}-\mathrm{F}_{20}\right.$ and $\left.\mathrm{F}_{20}-\mathrm{C}_{19}-\mathrm{C}_{16}-\mathrm{N}_{13}\right)$ and cis $\left(\mathrm{F}_{23}-\mathrm{C}_{22}-\right.$ $\mathrm{C}_{25}=\mathrm{O}_{26}$ ) arrangements (see Figure 6). Table 5 summarizes the IQF decomposition of the HF-D3/cc-pVTZ relative energies involving a four-fragment $O-P-Q-R$ partitioning in which fragments $P$ and $Q$ correspond to the central $\mathrm{C}_{22} \mathrm{HF}$ and $\mathrm{C}_{19} \mathrm{HF}$ units, respectively, fragment $O$ comprises the Ace- $\mathrm{CH}(\mathrm{Ph})-\mathrm{NHCO}$ - residue and fragment $R$ contains the isoindole-1,3dione moiety. The relative energies are now expressed as $\Delta E=E_{14 a-c o n f l}-E_{14 a-c o n f i}$ where $i=2$, 5 , or 6 . 
Figure 5. Ball-and-stick representation of selected conformers optimized for 14a at the HF-D3/cc-pVTZ level of theory. F-C-C-F, F-C-C-N, and F-C-C-C=O dihedral angles $\left(^{\circ}\right)$ and selected interatomic distances $(\AA)$ measured in the HF-D3/cc-pVTZ gas phase optimized structures are included. Energy differences $\left(E_{14 a-c o n f 1}-E_{14 a-c o n f i}\right)$ in $\mathrm{kcal} / \mathrm{mol}$ computed at the HF-D3/cc-pVTZ level of theory in the gas-phase and in chloroform (in Italics) are also included.

14a-conf1 $\Delta E=0.00 \quad 0.00$

14a-conf2 $\Delta E=0.74-1.05$

We first analyze the variation of the $\mathrm{F}_{23}-\mathrm{C}_{22}-\mathrm{C}_{19}-\mathrm{F}_{20}$ dihedral from $67.5^{\circ}$ in conformer 14a-conf1 to $-58.4^{\circ}$ in $14 \mathrm{a}-$ conf2, affecting the relative positioning of the $O$ and $R$ fragments (see Figure 5). As the two conformers are presumably stabilized by a similar gauche effect and the placement of the terminal isoindole-1,3-dione group in $R$ with reference to the central $P-Q$ fragments has little influence, their energy difference in the gas-phase is quite small, 0.7 $\mathrm{kcal} / \mathrm{mol}$ at the HF-D3/cc-pVTZ level favoring 14a-conf2. Nonetheless, examination of the IQF terms collected in Table 4 reveals some fine details concerning this energy difference. On one hand, there are some indications about a more stabilizing gauche effect in 14a-conf2 given that $\Delta E_{\mathrm{int,xc}}^{P \cdots}+\Delta E_{\mathrm{int}, \text { class }}^{P \ldots Q}=1.5-0.9=0.6 \mathrm{kcal} / \mathrm{mol}$. On the other one, IQF reveals substantial energy compensation occurring upon the formation of new $O \cdots R$ contacts with simultaneous 
loss of $P \cdot R$ and $Q \cdots R$ ones. Thus, the exchange-correlation interaction between fragments $O$ and $R$ largely stabilizes $14 \mathrm{a}-$ conf2 $\left(17.6 \mathrm{kcal} / \mathrm{mol}\right.$, comprising specific $\mathrm{H}_{17} \cdots \mathrm{O}_{26}=5.0 \mathrm{kcal} / \mathrm{mol}$, $\mathrm{C}_{16} \cdots \mathrm{O}_{26}=3.1, \mathrm{O}_{12} \cdots \mathrm{H}_{42}=3.0$ and $\mathrm{O}_{12} \cdots \mathrm{H}_{44}=2.3$ pair interactions; see Figure 5 and Table S11). This attractive component is partly neutralized by the equivalent terms accounting for the $O \cdots Q$ and $P \cdots R$ fragment interactions, which are -7.6 and $-4.4 \mathrm{kcal} / \mathrm{mol}$, respectively, reflecting the loss of the $\mathrm{F}_{20} \cdots \mathrm{H}_{44}$ and $\mathrm{H}_{21} \cdots \mathrm{O}_{26}$ contacts $(-3.2$ and $-2.2 \mathrm{kcal} / \mathrm{mol})$ and the $\mathrm{F}_{23} \cdots \mathrm{H}_{18}$ and $\mathrm{F}_{23} \cdots \mathrm{C}_{16}$ ones $(-2.0$ and $-1.8 \mathrm{kcal} / \mathrm{mol})$. In addition, the shortening of the $O \cdots R$ distance in 14a-conf2 implies not only stabilizing inter-fragment energy contributions, but also a large intra-fragment distortion as in the case of intermolecular complexes. For example, the $\Delta E_{n e t}^{R}$ term destabilizes $14 a-c o n f 2$ by $9.7 \mathrm{kcal} / \mathrm{mol}$. Altogether, these IQA components allow us to energetically weigh the formation/loss of intramolecular contacts upon the 14a-conf1 $\rightarrow$ 14aconf 2 transition, which nearly compensate each other.

Table 4. IQF energy components at the HF-D3/cc-pVTZ level for the energy difference $(\mathrm{kcal} / \mathrm{mol})$ among selected conformers optimized for compound 14a. $\mathrm{A}$ four-fragment partitioning scheme $(O=$ $\mathrm{CH}_{3} \mathrm{OCOCH}\left(\mathrm{CH}_{2} \mathrm{Ph}\right) \mathrm{NHCO}-, P=-\mathrm{CHF}-, Q=-\mathrm{CHF}-$, and $R=-\mathrm{CH}_{2}$-isoindol-1,3-dione) is assumed for the different conformers. Relative energies are given as $E_{14 a-c o n f l}-E_{14 a-c o n f i}$ so that a negative value means further stabilization of $14 a-$-conf1.

\begin{tabular}{|c|c|c|c|c|c|c|c|c|c|c|c|c|c|c|}
\hline & \multirow[t]{3}{*}{$\Delta E_{\text {net }}^{o}$} & \multirow[t]{3}{*}{$\Delta E_{n e t}^{P}$} & \multirow[t]{3}{*}{$\Delta E_{n e t}^{Q}$} & \multirow[t]{3}{*}{$\Delta E_{n e t}^{R}$} & \multicolumn{3}{|c|}{$\Delta E_{\text {int,disp }}$} & \multicolumn{2}{|r|}{$\Delta E_{\text {int }, x c}$} & \multicolumn{3}{|c|}{$\Delta E_{\text {int,class }}$} & \multirow[t]{3}{*}{$\Delta E_{I Q A}$} & \multirow[t]{3}{*}{$\Delta E_{H F-D 3}$} \\
\hline & & & & & $P \cdots Q$ & $P \cdots R$ & $o \cdots P$ & $P \cdots Q$ & $P \cdots R \quad O \cdots P$ & $P \cdots Q$ & $P \cdots R$ & $o \cdots P$ & & \\
\hline & & & & & $Q \cdots R$ & $O \cdots Q$ & $O \cdots R$ & $Q \cdots R$ & $O \cdots Q \quad O \cdots R$ & $Q \cdots R$ & $o \cdots Q$ & $O \cdots R$ & & \\
\hline \multirow[t]{2}{*}{$\Delta E=E_{\text {14a-confl } 1}-E_{\text {14a-conf } 2}$} & -5.5 & -1.2 & 2.8 & -9.7 & 0.3 & -0.8 & 0.1 & 1.5 & $-4.4,1.6$ & -0.9 & -1.1 & -0.8 & 0.3 & 0.7 \\
\hline & & & & & 0.2 & -0.9 & 4.5 & 0.2 & $-7.6, \quad 17.6$ & 0.8 & -1.0 & 4.7 & & \\
\hline \multirow[t]{2}{*}{$\Delta E=E_{14 a-c o n f l}-E_{14 a-c o n f 5}$} & -17.5 & -1.0 & 4.5 & -19.8 & 0.3 & -0.9 & 0.1 & -2.5 & $-4.5, \quad 2.2$ & 2.8 & -1.2 & 0.5 & -2.2 & -2.0 \\
\hline & & & & & -0.4 & -1.8 & 12.5 & -0.5 & $-9.6,35.2$ & -0.2 & -3.2 & 2.8 & & \\
\hline \multirow[t]{2}{*}{$\Delta E=E_{14 a-c o n f l}-E_{14 a-c o n f 6}$} & -16.9 & -3.4 & 3.2 & -21.7 & 0.3 & 0.7 & -0.4 & -1.5 & $1.1,-2.4$ & 2.5 & -0.1 & -6.7 & -2.1 & -2.6 \\
\hline & & & & & -0.7 & -0.8 & 10.8 & -4.6 & $-4.9,33.7$ & 2.0 & 1.1 & 6.5 & & \\
\hline
\end{tabular}

More interesting conformational effects act upon 14a-conf5, whose overall shape differs significantly with respect to those of $14 a$-conf1/14a-conf2. There is an anti $\mathrm{F}_{23}-\mathrm{C}_{22}-$ $\mathrm{C}_{19}-\mathrm{F}_{20}$ arrangement in 14a-conf5, which is $2.0 \mathrm{kcal} / \mathrm{mol}$ less stable than $14 a-c o n f 1$ at $\mathrm{HF}$ D3/cc-pVTZ. However, this energy difference cannot be assigned to the loss of the gauche $\mathrm{F}_{23}-\mathrm{C}_{22}-\mathrm{C}_{19}-\mathrm{F}_{20}$ effect, because the IQF exchange-correlation term favoring the gauche orientation $\left(\Delta E_{\mathrm{int}, \mathrm{xc}}^{P \cdots Q}=-2.5 \mathrm{kcal} / \mathrm{mol}\right)$ is completely canceled out by the electrostatic term ( $\Delta E_{\text {int,class }}^{P \cdots Q}=2.7 \mathrm{kcal} / \mathrm{mol}$ ) favoring the anti one. Likewise the $g+/ g+$ and anti/anti pair in 11, the exchange-correlation stabilization of the gauche F-C-C-F arrangement in 14a-conf1 is reversed by the electrostatic term favoring the anti orientation in 14a-conf5, what is in contrast with the results obtained for the small models 1-5. Hence, the destabilization of 14a- 
conf5 with reference to $14 a-c o n f 1$ is better rationalized in terms of the forming/breaking of intramolecular contacts. Thus, the intermediate distance ( $5.1 \AA)$ between the center of mass of the aromatic rings in fragments $O$ and $R$ with an almost parallel arrangement of the ring planes $\left(12.1^{\circ}\right)$ may allow some $\pi-\pi$ favorable interaction in 14a-conf5, whereas the loss of the $\mathrm{CH} \cdots \mathrm{F}$ contacts characteristic of 14a-conf1 would disfavor it. The energetic impact of these structural changes can be assessed through the IQF components. One the one hand, the approaching of the terminal $O \cdots R$ groups gives large and stabilizing values for the $\Delta E_{\text {int,disp }}^{O \cdots R}$ $(12.5 \mathrm{kcal} / \mathrm{mol})$ and $\Delta E_{\mathrm{int}, \mathrm{xc}}^{O \cdots R}(35.2 \mathrm{kcal} / \mathrm{mol})$ terms. The $\Delta E_{\mathrm{int}, \mathrm{xc}}^{O \cdots R}$ value stems from many interatomic contributions involving the $-\mathrm{CH}_{2}-\mathrm{N}$ moiety in fragment $R$ and the amide and phenyl groups in fragment $O\left(\right.$ e.g., $\mathrm{H}_{18} \cdots \mathrm{O}_{26} 3.6 \mathrm{kcal} / \mathrm{mol}, \mathrm{N}_{13} \cdots \mathrm{C}_{39} 2.6 \mathrm{kcal} / \mathrm{mol}, \mathrm{C}_{16} \cdots \mathrm{O}_{26} 2.2$ $\mathrm{kcal} / \mathrm{mol}, \mathrm{H}_{17} \cdots \mathrm{C}_{35} 2.1 \mathrm{kcal} / \mathrm{mol}$, etc.). The closer $O \cdots R$ contacts are accompanied by opposite intra-fragment distortion effects for the $O$ and $R$ moieties $(-17.5$ and $-19.8 \mathrm{kcal} / \mathrm{mol})$ so that the sum $\Delta E_{\text {net }}^{O}+\Delta E_{\text {net }}^{R}+\Delta E_{\mathrm{int}, \mathrm{disp}}^{O \cdots R}+\Delta E_{\mathrm{int}, x c}^{O \cdots R}$ amounts to $10.4 \mathrm{kcal} / \mathrm{mol}$ favoring 14a-conf5. On the other hand, this contribution is overcompensated by the penalty associated with the loss of the contacts exhibited by $14 a-c o n f 1\left(\mathrm{C}_{16}-\mathrm{H}_{18} \cdots \mathrm{F}_{23}, \mathrm{~F}_{20} \cdots \mathrm{H}_{44}\right.$, and $\mathrm{H}_{21} \cdots \mathrm{O}_{26}$, see Figure 6) that is mainly accounted for by $\Delta E_{\mathrm{int}, \mathrm{xc}}^{P \cdots R}=-4.5$ and $\Delta E_{\mathrm{int}, \mathrm{xc}}^{O \cdots Q}=-9.6 \mathrm{kcal} / \mathrm{mol}$, determining thus the lower stability of $14 a-c o n f 5$.

As previously noticed, the cis $\mathrm{F}-\mathrm{C}-\mathrm{C}=\mathrm{O}$ arrangement in $\mathbf{1 0}$ or $\mathbf{1 2}$ implies a large destabilization of more than $5 \mathrm{kcal} / \mathrm{mol}$ with respect to the trans orientation, basically due to the dipole-dipole interaction between the $\mathrm{F}-\mathrm{C}$ and $\mathrm{C}=\mathrm{O}$ groups. We see in Figure 6 that 14aconf6 has a cis $\mathrm{F}_{23}-\mathrm{C}_{22}-\mathrm{C}_{25}=\mathrm{O}_{26}$ orientation and two anti $\mathrm{F}_{23}-\mathrm{C}_{22}-\mathrm{C}_{19}-\mathrm{F}_{20}$ and $\mathrm{F}_{20}-\mathrm{C}_{19}-\mathrm{C}_{16}-\mathrm{N}_{13}$ groups, but it is only $2.6 \mathrm{kcal} / \mathrm{mol}$ less stable than 14a-conf1. Nevertheless, the impact of the cis $\mathrm{F}_{23}-\mathrm{C}_{22}-\mathrm{C}_{25}=\mathrm{O}_{26}$ dihedral in the electrostatic $\Delta E_{\text {int,class }}^{O \ldots P}$ value is $-6.7 \mathrm{kcal} / \mathrm{mol}$, which is similar to those observed in $\mathbf{1 0}$ or $\mathbf{1 2}$. The gauche effect is again reversed in the 14a-conf1/14aconf6 pair as the sum $\Delta E_{\mathrm{int}, \mathrm{xc}}^{P \cdots Q}+\Delta E_{\mathrm{int,class}}^{P \cdots Q}$ equals to $1.0 \mathrm{kcal} / \mathrm{mol}$. Further stabilization of 14aconf6 comes from the various $O \cdots R$ interactions between the bulky terminal fragments, partially compensating the unfavorable cis $\mathrm{F}-\mathrm{C}-\mathrm{C}=\mathrm{O}$ orientation. These interactions can be measured in terms of the sum $\Delta E_{\text {net }}^{O}+\Delta E_{\text {net }}^{R}+\Delta E_{\mathrm{int,disp}}^{O \cdots R}+\Delta E_{\mathrm{int}, x c}^{O \cdots R}=5.9 \mathrm{kcal} / \mathrm{mol}$. In addition, only one of the two $\mathrm{CH} \cdots \mathrm{F}$ contacts is lost upon the 14a-conf1 $\rightarrow$ 14a-conf6 transition, determining an energy change $\Delta E_{\mathrm{int}, \mathrm{xc}}^{P \cdots R}+\Delta E_{\mathrm{int,xc}}^{O \cdots Q}=-3.8 \mathrm{kcal} / \mathrm{mol}$, which does not neutralize the global effect of the $O \cdots R$ contacts. Therefore, IQF shows how the a priori strong preference for the trans 
F-C-C=O conformation in the $14 a-c o n f 1 / 14 a-c o n f 6$ pair is significantly reduced by the unexpected reversal of the gauche effect and the corresponding balance of interactions between the terminal groups.

\section{Discussion and Conclusions}

Among the major conformational effects associated with $\mathrm{C}-\mathrm{F}$ bonds, the gauche preference in $\mathrm{F}-\mathrm{C}-\mathrm{C}-\mathrm{X}$ moieties $(\mathrm{X}=\mathrm{F}, \mathrm{O}, \mathrm{N}$, or $\mathrm{C}$ ), the repulsion between $\mathrm{C}-\mathrm{F}$ bonds aligned parallel in 1,3-positions, and the favored trans-planar arrangement in $\mathrm{F}-\mathrm{C}-\mathrm{C}=\mathrm{O}$ moieties, are usually invoked to rationalize or foresee conformations in fluorine containing systems. However, either X-ray structures or NMR measurements have revealed molecular conformations that differ from those expected according to the usual fluorine effects. ${ }^{15}$ Moreover, two possible gauche orientations $\left(+60 \%-60^{\circ}\right)$ are accessible to $\mathrm{F}-\mathrm{C}-\mathrm{C}-\mathrm{X}$ moieties, which in most cases result in different molecular conformations. Hence, additional intramolecular interactions and environmental effects may play a significant role in order to explain the conformational preferences of fluorinated molecules. To gain further understanding of such effects, IQA, as a reference-free energy decomposition method, can be useful to express the conformational energies predicted by QM calculations into unambiguously contributions, which include electrostatic and exchange-correlation interatomic interactions.

Concerning the prototypical gauche effect in 1,2-difluoroethane, the IQA method has been formerly employed ${ }^{24}$ to highlight specifically the gauche stabilization due to the 1,3 Coulombic attraction between $\mathrm{C}$ and $\mathrm{F}$ atoms. However, we note that there are strong $\mathrm{F} \cdots \mathrm{F}$ and $\mathrm{C} \cdots \mathrm{C}$ repulsions as characterized by IQA that can be seen to compensate the $\mathrm{C} \cdots \mathrm{F}$ attractive energies. Moreover, the larger $\mathrm{C}-\mathrm{C}-\mathrm{F}$ angles and $\mathrm{C} \cdots \mathrm{F}$ distances obtained in the gauche form as compared to the anti one (110.3/108.1을 and 2.34/2.32 $\AA$ ) seem not compatible with explaining the gauche effect as the consequence of the 1,3 electrostatic attraction between $\mathrm{C}$ and $\mathrm{F}$ atoms. In addition, we also consider that other terms like the $\mathrm{C} \cdots \mathrm{C}$ and $\mathrm{C} \cdots \mathrm{F}$ exchange-correlation interactions contribute to the gauche stability. Thus, when the IQA atomic terms are grouped by defining two interacting $\mathrm{CH}_{2} \mathrm{~F}$ fragments separated by the rotatable bond, it emerges that the gauche structure is stabilized by the exchange-correlation interaction and destabilized by the electrostatic $\mathrm{CH}_{2} \mathrm{~F} \cdots \mathrm{CH}_{2} \mathrm{~F}$ tem, involving only a minor distortion at each fragment. In this fragment-based IQF analysis, the electrostatic interaction can be further decomposed to show how the gauche destabilization is partially due to dipole-dipole repulsion, the dipoles being mainly associated to the C-F 
bonds. We believe that the IQF interpretation of the gauche effect in 1,2-difluoroethane is more chemically appealing and, accordingly, we apply it to the rest of molecules considered in this work. We also note that, regardless of atomic contributions being grouped or not, IQA clearly shows that both electrostatic and exchange-correlation interactions should be considered to explain the gauche effect in 1,2-difluorethane.

Examination of the 1,2-difluoroethane related systems 2-7 bearing different polar/charged substituents points out that the quantum exchange-correlation inter-fragment interaction systematically stabilizes the gauche conformation. In contrast, the electrostatic interaction term is system-dependent because the sign and magnitude of $\Delta E_{\text {int,class }}^{P \cdots Q}$ is mainly determined by the particular interaction(s) between the fluorine atom in fragment $P$ and the closest atoms in fragment $Q$. For the positively charged systems, the exchange-correlation and the classical electrostatic inter-fragment interactions are large and comparable, which contrasts with previous assumptions explaining their gauche preference only in terms of electrostatic effects. IQF also emphasizes that the magnitude and nature of the electrostatic interactions depends on the particular characteristics of the charged moiety.

As previously proposed, ${ }^{9}$ dipole-dipole interaction arises as the main contribution to the stability of the trans-planar $\mathrm{F}-\mathrm{C}-\mathrm{C}=\mathrm{O}$ arrangement in the $\mathrm{CH}_{2} \mathrm{~F}-\mathrm{COX}$ molecules (8-10). However, the exchange-correlation term is also relevant for the ketone (8) and the amide (10) derivatives. More particularly, the interatomic F...HN interaction in $\mathbf{1 0}$ makes a large contribution to the $\Delta E_{\mathrm{int}, \mathrm{xc}}^{P \cdots Q}$ term. This relatively large exchange-correlation interaction between fluorine and nearby hydrogen atoms, also observed in 3-fluoropropanal (3) and 2-fluoroethan1-aminium (6), can be considered as the signature for a (weak) hydrogen bond. Fluorine is considered a poor hydrogen bonding acceptor although there are examples of crystal structures showing short intramolecular contacts between organic fluorine and $\mathrm{HO}-/ \mathrm{HN}$ moieties $^{9,57}$ that have been assumed to result almost exclusively from dipole-dipole electrostatic interactions, excepting for $\mathrm{F} \cdots \mathrm{HC}$ contacts where it is thought that dispersion also plays a role. Nonetheless, our calculations help clarify the nature and impact of these $\mathrm{F} \cdots \mathrm{HN}$ and $\mathrm{F} \cdots \mathrm{HC}$ contacts that particularly affect the quantum mechanical interaction between the $\mathrm{H}$-bonded groups, in line with recent ${ }^{4} \mathrm{JHF}$ measurements and NBO calculations performed for $\alpha$-fluoro amides. ${ }^{30}$ Thus, we conclude that the largest exchange-correlation IQA energies help identify the relevant $\mathrm{F} \cdots \mathrm{HC} / \mathrm{HN}$ interactions. 
To analyze the various fluorine effects in more complex settings, we examined all the possible conformations in 1,2,3-trifluoropropane (11) and two other compounds (12 and 13) related to $\mathbf{1 4 a / 1 4 b}$. The two less stable conformers obtained for 1,2,3-trifluoropropane present the unfavorable parallel alignment of 1,3 C-F bonds previously described in polyfluorinated alkanes. ${ }^{9}$ IQF confirms the appearance of an unfavorable electrostatic interaction between the $1,3 \mathrm{CH}_{2} \mathrm{~F}$ groups and ascribes it to dipole-dipole repulsion. But unexpectedly, the most stable conformer obtained for 1,2,3-trifluoropropane does not result from maximizing the number of gauche effects and minimizing the 1,3 dipole-dipole repulsions. According to our analyses, the presence of $1,3 \mathrm{~F} \cdots \mathrm{H}-\mathrm{C}$ interactions also contributes to explain the relative stability of the conformers. These $\mathrm{F} \cdots \mathrm{H}-\mathrm{C}$ contacts are among the largest exchange-correlation interatomic interactions in 1,2,3-trifluoropropane and their stabilizing effect is larger than the gauche effect. The partial alignment of the amide dipole with a $\mathrm{C}-\mathrm{F}$ bond and the presence of different $\mathrm{F} \cdots \mathrm{H}$ contacts also explain the conformational landscape obtained for 2,3-difluoro- $N$-methylpropanamide (12).

The various analyses carried out on the model systems may help us to better ascertain the various effects determining the conformational properties of the difluorinated dipeptide 14a and 14b diastereomers. According to X-ray crystallography, the $(R, R)$ or $(R, S)$ stereochemistry for the fluorination at the central $\mathrm{C}_{22}$ and $\mathrm{C}_{19}$ atoms leads to a different backbone conformation for the whole molecule. ${ }^{14}$ In the two crystal structures, the vicinal $\mathrm{C}_{22}-$ $\mathrm{F}_{23} / \mathrm{C}_{19}-\mathrm{F}_{20}$ and $\mathrm{C}_{19}-\mathrm{F}_{20} / \mathrm{C}_{16}-\mathrm{N}_{13}$ bonds are in gauche while the $\mathrm{C}_{22}-\mathrm{F}_{23}$ bond aligns antiparallel to the adjacent amide carbonyl, which seems in consonance with expectations. The QMrefined conformational search performed for $14 a / 14 b$ predicts that the lowest energy conformer either in the gas-phase or in the solvent-continuum is structurally close to the crystallographic structure. This seems to indicate that crystal packing and solvent effects would play only a minor role in the conformational preferences of $\mathbf{1 4 a / 1 4 b}$, the intramolecular factors being dominant. However, the conformational search illustrates that the observed crystal structures are not the only conformers compatible with the effects usually assigned to fluorine atoms. For instance, two alternative gauche forms $(g+$ or $g$-) could be accessible for the vicinal $\mathrm{C}_{22}-\mathrm{F}_{23} / \mathrm{C}_{19}-\mathrm{F}_{20}$ and $\mathrm{C}_{19}-\mathrm{F}_{20} / \mathrm{C}_{16}-\mathrm{N}_{13}$ bonds in the gas-phase or in solution. This seems confirmed by our estimations of the ${ }^{3} J_{H F}$ values in chloroform obtained for the vicinal fluorine and hydrogen atoms bound to $\mathrm{C}_{22} / \mathrm{C}_{19}$ in $\mathbf{1 4 a} / \mathbf{1 4 b}$, which are in reasonable agreement with experimental data. Moreover, the energetic penalty associated to the presence of unfavorable anti or cis arrangements around the fluorine atoms is not dramatic (e.g., 2.5 
$\mathrm{kcal} / \mathrm{mol}$ ), pointing thus towards the compensating roles of other stabilizing intra-molecular interactions.

The IQA energy decomposition can treat relatively large molecules like the selected conformers of $14 \mathrm{a}$. Three of them differ in the $g_{+}, g$-, or anti arrangement of the $\mathrm{F}_{23}-\mathrm{C}_{22}-\mathrm{C}_{19-}$ $F_{20}$ moiety, whereas the fourth one presents the less favorable $\mathrm{F}_{23}-\mathrm{C}_{22}-\mathrm{C}_{19}-\mathrm{F}_{20}$ and $\mathrm{F}_{20}-\mathrm{C}_{19}-$ $\mathrm{C}_{16}-\mathrm{N}_{13}$ anti and $\mathrm{F}_{23}-\mathrm{C}_{22}-\mathrm{C}_{25}=\mathrm{O}_{26}$ cis orientations. In all the cases, the careful examination of the IQF descriptors allows us to assess the energetic impact of the gauche/anti and cis/trans effects. In comparison with the smaller model systems 8-10 and 12, IQF indicates that the important electrostatic $\mathrm{F}-\mathrm{C}-\mathrm{C}=\mathrm{O}$ trans preference $(\sim 6-8 \mathrm{kcal} / \mathrm{mol})$ is appreciably transferable. Interestingly, the exchange-correlation and electrostatic balance favoring the gauche F-C-C$\mathrm{F}$ orientation can be altered in some of the $\mathbf{1 4 a}$ conformers (also in $\mathbf{1 1}$ conformers) resulting in a small inverted gauche effect $(<1.0 \mathrm{kcal} / \mathrm{mol})$ that benefits the anti $\mathrm{F}-\mathrm{C}-\mathrm{C}-\mathrm{F}$ arrangement. Furthermore, our four-fragment IQA partitioning points out that the formation/rupture of other intramolecular contacts (e.g., $\pi \cdots \pi, \mathrm{F} \cdots \mathrm{H}-\mathrm{C}$, etc.) can modulate and/or attenuate the F-C$\mathrm{C}=\mathrm{O}$ trans and the F-C-C-F gauche effects. Therefore, our results stress that the gauche effect can be both system and conformation dependent, what is in consonance with previous studies ${ }^{19,26-28}$ showing that environmental (solvent) effects can modulate or alter the gauche conformational preference.

In summary, our QM calculations complemented with the HF-D3 IQF energy decompositions are useful to analyze in a systematic and consistent manner the energetic preferences of small fluorinated compounds having either gauche/anti or cis/trans conformations. Following the IQF approach, we find a clear correspondence between specific exchange-correlation and/or electrostatic fragment-interaction energies with the appearance of the gauche/anti or cis/trans effects, providing also insight into their magnitude and nature. The same IQF approach can be extended to assess those gauche/anti or cis/trans effects in molecules with two or more rotatable bonds as well as to study the roles played by other concomitant effects (e.g., specific $\mathrm{CH} / \mathrm{OH} / \mathrm{NH} \cdots \mathrm{F}$ contacts, $1-3$ electrostatic interactions, etc.). For the relatively large $\alpha, \beta$-difluoro- $\gamma$-amino acid derivatives, our conformational search followed by QM and selected IQF calculations complement well their crystallographic and NMR characterization. The conformational preferences of these compounds as detailed by the theoretical analysis point out that the gauche/anti and cis/trans effects associated to fluorine bonds may be attenuated in large molecules, where the most preferred conformations may be dictated by other non-fluorine specific intra-molecular interactions. 
Finally, we note that similar computational protocols including solvent effects could help in the rational design of fluorinated molecules having a nearly frozen conformation tailored for a particular application. This is still a challenging task as most of the fluorinated compounds have emerged from broad chemical screening programs and the actual influence of fluorine is considered retrospectively. ${ }^{9}$ However, the QM and IQF assessments of the conformational effects associated to the presence of fluorine atoms (gauche effect, the 1,3 C-F repulsion and other electrostatic interactions, the hydrogen bond acceptor capability of organic fluorine, etc.) could result in new guidelines to predict the conformation of structurallycomplex fluorinated molecules.

\section{Acknowledgments}

This research was supported by the CTQ2015-65790-P (MICINN, Spain) and the IDI/2018/000177 (FICYT, Spain) grants. F. J.-G. gratefully acknowledges financial support from the MICINN (grant BES-2016-076986). The authors are grateful to J.L. Casals-Sainz for his assistance in the correlated IQA calculations.

\section{Electronic Supplementary Information}

Table S1 with the sum of the different IQA atomic contribution for the small (1-10) models. Tables S2-S5 and S11 with the largest changes in the IQA atomic contributions for the different systems. Figure S1 with a representation of the different conformers obtained for 13 and their relative energies. Table S6 and S7 with IQF and the largest changes in the IQA atomic contributions and results obtained for 13. Tables S8 and S9 with energies and geometrical parameters obtained for the different conformers optimized for $\mathbf{1 4 a}$ and $\mathbf{1 4 b}$. Figures S2 and S3 with a ball-and-stick representation of the different conformers optimized for $14 a$ and $14 b$. Table $S 10$ with the ${ }^{3} \mathrm{~J}_{\mathrm{H}, \mathrm{F}}$ coupling constants estimated for some of the $14 a$ and 14b conformers. Table S12 with the equivalence between the filenames with the Cartesian coordinates (COORD.zip) and the HF/MP2 optimized compounds. Table S13 comparing the HF-D3 IQF and correlated IQF results for 1,2-difluoroethane. 


\section{References}

1. Y. Zhou, J. Wang, Z. Gu, S. Wang, W. Zhu, J. L. Aceña, V. A. Soloshonok, K. Izawa and H. Liu, Chem. Rev., 2016, 116, 422-518.

2. P. Jeschke, ChemBioChem, 2004, 5, 570-589.

3. T. Fujiwara and D. O'Hagan, J. Fluor. Chem., 2014, 167, 16-29.

4. $\quad$ P. Kirsch, J. Fluor. Chem., 2015, 177, 29-36.

5. J. H. Yun, S. Park, J. H. Heo, H.-S. Lee, S. Yoon, J. Kang, S. H. Im, H. Kim, W. Lee, B. Kim, M. J. Ko, D. S. Chung and H. J. Son, Chem. Sci., 2016, 7, 6649-6661.

6. L. E. Zimmer, C. Sparr and R. Gilmour, Angew. Chem. Int. Ed., 2011, 50, 1186011871.

7. D. Cahard and V. Bizet, Chem. Soc. Rev., 2014, 43, 135-147.

8. A. A. Berger, J.-S. Völler, N. Budisa and B. Koksch, Acc. Chem. Res., 2017, 50, 20932103.

9. D. O'Hagan, Chem. Soc. Rev., 2008, 37, 308-319.

10. H.-J. Böhm, D. Banner, S. Bendels, M. Kansy, B. Kuhn, K. Müller, U. Obst-Sander and M. Stahl, ChemBioChem, 2004, 5, 637-643.

11. J. W. Banks, A. S. Batsanov, J. A. K. Howard, D. O'Hagan, H. S. Rzepa and S. MartinSantamaria, J. Chem. Soc., Perkin Trans. 2, 1999, 2409-2411.

12. M. Schüler, D. O'Hagan and A. M. Z. Slawin, Chem. Commun., 2005, 4324-4326.

13. L. Hunter, P. Kirsch, A. M. Z. Slawin and D. O'Hagan, Angew. Chem., 2009, 121, 5565-5568.

14. L. Hunter, K. A. Jolliffe, M. J. T. Jordan, P. Jensen and R. B. Macquart, Chem. Eur. J., 2011, 17, 2340-2343.

15. F. Scheidt, P. Selter, N. Santschi, M. C. Holland, D. V. Dudenko, C. Daniliuc, C. MeckLichtenfeld, M. R. Hansen and R. Gilmour, Chem. Eur. J., 2017, 23, 6142-6149.

16. N. E. J. Gooseman, D. O'Hagan, M. J. G. Peach, A. M. Z. Slawin, D. J. Tozer and R. J. Young, Angew. Chem., 2007, 119, 6008-6012.

17. L. Goodman, H. Gu and V. Pophristic, J. Phys. Chem. A, 2005, 109, 1223-1229.

18. D. Y. Buissonneaud, T. van Mourik and D. O'Hagan, Tetrahedron, 2010, 66, 21962202.

19. F. A. Martins and M. P. Freitas, Eur. J. Org. Chem., 2019, 6401-6406.

20. F. Weinhold, C. R. Landis and E. D. Glendening, Int. Rev. Phys. Chem., 2016, 35, 399-440. 
21. F. M. Bickelhaupt and E. J. Baerends, Angew. Chem. Int. Ed., 2003, 42, 4183-4188.

22. P. Su and H. Li, J. Chem. Phys., 2009, 131, 014102.

23. M. Baranac-Stojanović, RSC Adv., 2014, 4, 43834-43838.

24. J. C. R. Thacker and P. L. A. Popelier, J. Phys. Chem. A, 2018, 122, 1439-1450.

25. C. R. S. Briggs, M. J. Allen, D. O'Hagan, D. J. Tozer, A. M. Z. Slawin, A. E. Goeta and J. A. K. Howard, Org. Biomol. Chem., 2004, 2, 732-740.

26. C. Tormena, M. P. Freitas, R. Rittner and R. J. Abraham, Phys. Chem. Chem. Phys., 2004, 6, 1152-1156.

27. B. C. Fiorin, E. A. Basso, C. Tormena, R. Rittner and R. J. Abraham, J. Phys. Chem. A, 2009, 113, 2906-2913.

28. G. Pattison, Beilstein J. Org. Chem., 2017, 13, 2915-2921.

29. C. Trindle, P. Crum and K. Douglass, J. Phys. Chem. A, 2003, 107, 6236-6242.

30. E. Cosimi, N. Trapp, M.-C. Ebert and H. Wennemers, Chem. Commun., 2019, 55, 2253-2256.

31. J. Aleksić, M. Stojanović and M. Baranac-Stojanović, J. Org. Chem., 2015, 80, 1019710207.

32. E. Bogdan, G. Compain, L. Mtashobya, J.-Y. Le Questel, F. Besseau, N. Galland, B. Linclau and J. Graton, Chem. Eur. J., 2015, 21, 11462-11474.

33. R. A. Cormanich, D. O'Hagan and M. Bühl, Angew. Chem. Int. Ed., 2017, 56, 78677870.

34. M. A. Blanco, A. Martín Pendás and E. Francisco, J. Chem. Theory Comput., 2005, 1, 1096-1109.

35. D. Suárez, N. Díaz, E. Francisco and A. Martín Pendás, ChemPhysChem, 2018, 19, 973-987.

36. S. Grimme, WIREs Comput Mol Sci, 2011, 1, 211-228.

37. A. D. Becke and E. R. Johnson, J. Chem. Phys., 2005, 122, 154104.

38. L. Goerigk and J. R. Reimers, J. Chem. Theory Comput., 2013, 9, 3240-3251.

39. L. Goerigk, C. A. Collyer and J. R. Reimers, J. Phys. Chem. B, 2014, 118, 1461214626.

40. T. Ziegler and A. Rauk, Theoret. Chim. Acta, 1977, 46, 1-10.

41. F. M. Bickelhaupt and E. J. Baerends, in Rev. Comput. Chem., John Wiley \& Sons, Inc., 2007, DOI: 10.1002/9780470125922.ch1, pp. 1-86. 
42. E. Francisco and A. Martín Pendás, in Non-Covalent Interactions in Quantum Chemistry and Physics, eds. A. Otero de la Roza and G. A. DiLabio, Elsevier, 2017, DOI: https://doi.org/10.1016/B978-0-12-809835-6.00003-7, pp. 27-64.

43. C. Outeiral, M. A. Vincent, Á. Martín Pendás and P. L. A. Popelier, Chemical Science, 2018, 9, 5517-5529.

44. E. F. Pettersen, T. D. Goddard, C. C. Huang, G. S. Couch, D. M. Greenblatt, E. C. Meng and T. E. Ferrin, J Comput Chem., 2004, 25, 1605-1612.

45. F. Neese, WIREs Comput Mol Sci, 2012, 2, 73-78.

46. S. Grimme, A. Hansen, J. G. Brandenburg and C. Bannwarth, Chem. Rev., 2016, 116, 5105-5154.

47. D. G. Liakos, M. Sparta, M. K. Kesharwani, J. M. L. Martin and F. Neese, J. Chem. Theory Comput., 2015, 11, 1525-1539.

48. N. Sauton, D. Lagorce, B. O. Villoutreix and M. A. Miteva, BMC Bioinformatics, 2008, 9, 184.

49. J. Wang, W. Wang, P. A. Kollman and D. A. Case, J Mol Graph Model, 2006, 25, 247260.

50. A. V. Marenich, C. J. Cramer and D. G. Truhlar, J. Phys. Chem. B, 2009, 113, 63786396.

51. C. Thibaudeau, J. Plavec and J. Chattopadhyaya, J. Org. Chem., 1998, 63, 49674984.

52. A. M. Pendas and E. Francisco, Unpublished, 2015.

53. S. Grimme, J. Antony, S. Ehrlich and H. Krieg, J. Chem. Phys., 2010, 132, 154104.

54. E. Francisco, D. Menéndez Crespo, A. Costales and Á. Martín Pendás, J. Comput. Chem., 2017, 38, 816-829.

55. J. Pipek and P. G. Mezey, J. Chem. Phys., 1989, 90, 4916-4926.

56. A. Martín Pendás, M. A. Blanco and E. Francisco, J Comput Chem, 2009, 30, 98-109.

57. I. Hyla-Kryspin, G. Haufe and S. Grimme, Chem. Eur. J., 2004, 10, 3411-3422. 